\title{
化学链合成氨研究进展
}

\author{
冯圣 ${ }^{a, b}$ 高文波 ${ }^{a}$ 曹湖军 ${ }^{a}$ 郭建平*, $a$ 陈萍 $a, b$ \\ $\left({ }^{a}\right.$ 中国科学院大连化学物理研究所 中国科学院洁净能源创新研究院 能源材料化学协同创新中心(iChEM) \\ 大连 116023) \\ $\left(^{b}\right.$ 大连理工大学 张大显化学学院 大连 116024)
}

\begin{abstract}
摘要 氨不仅是氮肥的主要原料, 近年来亦被认为是一种具有重要应用前景的 “能源载体”. 现有的 Haber-Bosch 合成 氨工艺是一严重依赖化石能源、高能耗、高碳排放的过程. 开发可再生能源驱动的新型 “绿色” 合成氨过程是实现人 类社会可持续发展的重要课题. 将合成氨反应解耦为两个或多个分步反应, 即化学链合成氨过程, 具有易与可再生能 源耦合、常压操作、规避反应物竞争吸附等特点, 越来越受到业界的关注. 本综述阐述了化学链合成氨的发展历史及 近期研究进展, 重点总结了载氮体材料的研发现状, 并对高效载氮体的设计笁选以及化学链合成氨研究所面临的挑战 进行了分析和探讨.
\end{abstract}

关键词 合成氨; 化学链；载氮体；氮化物；亚氨基化合物

\section{Advances in the Chemical Looping Ammonia Synthesis}

\author{
Feng, Sheng ${ }^{a, b}$ Gao, Wenbo ${ }^{a}$ Cao, Hujun ${ }^{a} \quad$ Guo, Jianping*, ${ }^{*} \quad$ Chen, Ping ${ }^{a, b}$ \\ $\left({ }^{a}\right.$ Dalian National Laboratory for Clean Energy, Collaborative Innovation Center of Chemistry for Energy Materials, \\ Dalian Institute of Chemical Physics, Chinese Academy of Sciences, Dalian 116023, China) \\ ( ${ }^{b}$ Zhang Dayu School of Chemistry, Dalian University of Technology, Dalian 116024, China)
}

\begin{abstract}
Ammonia is not only the main raw material of nitrogen fertilizer, but also a promising energy carrier for the storage and utilization of renewable energy. The fossil fuel-based Haber-Bosch ammonia synthesis industry is an energy-consuming and high $\mathrm{CO}_{2}$-emission process. For the sustainable growth of human society, it is critically important to develop "green" ammonia synthesis processes driven by renewable energies. This scenario motivates growing interests on ammonia synthesis via heterogeneous catalysis, electro-chemical and photo-chemical routes as well as chemical looping process. Chemical looping ammonia synthesis (CLAS) process involves a series of individual reactions which produce ammonia in a distinctly different manner to the catalytic process. The CLAS could be operated under ambient pressure, and the switching on/off operation is flexible. Therefore, CLAS may be more amenable to variable and intermittent operation compared to the conventional catalytic process. More importantly, the competitive adsorption of $\mathrm{N}_{2}$ and $\mathrm{H}_{2}$ or $\mathrm{H}_{2} \mathrm{O}$ in the catalytic process can be circumvented to a great extent, which opens new opportunities for the design and development of nitrogen carriers especially for low-temperature ammonia production. Because of these unique features, the application of chemical looping technology for ammonia synthesis has been received increasing attention in recent years. The development of high-efficiency nitrogen carriers is the key component for the implementation of CLAS. A wide range of materials including metal nitrides, metal imides, nitride-hydrides and oxynitrides have been evaluated as nitrogen carriers for CLAS. The knowledge accumulated during the past decade will no doubt beneficial for the further optimization and development of nitrogen carriers. This article reviews the research progress in the field of chemical looping ammonia synthesis in recent years, with the focuses on the materials development of nitrogen carriers in CLAS. Furthermore, the challenges and future directions of CLAS are also discussed. With the development of nitrogen carriers and process design, CLAS would potentially play an important role in the green ammonia synthesis as well as the future energy system.
\end{abstract}

Keywords ammonia synthesis; chemical looping; nitrogen carrier; nitride; imide

\section{1 引言}

氨是氮肥的主要原料, 同时, 亦是人工合成几乎所 有重要含氮化学品的原料 ${ }^{[1]}$. 在合成氨工业建立一个世 纪后, 氨又因其能量密度高 $\left(3 \mathrm{kWh} \cdot \mathrm{kg}^{-1}\right)$ 、氢含量高(质
量分数 $17.7 \%$ )、易储运、相关基础设施成熟等特点而被 认为是重要的能源载体(Energy carrier) ${ }^{[2-5]}$. 目前, 可再 生能源的利用面临间歇性、波动性、并网发电难等问题, 寻找安全高效的能量存储方式是当前可再生能源利用 所面临的巨大挑战. 而将可再生能源通过合成氨反应储

* E-mail: guojianping@dicp.ac.cn

Received June 4, 2020; published July 9, 2020.

Project supported by the National Natural Science Foundation of China (Nos. 21872137, 21922205) and Youth Innovation Promotion Association Chinese Academy of Sciences (No. 2018213).

项目受国家自然科学基金(Nos. 21872137, 21922205)和中国科学院青年创新促进会(No. 2018213)资助. 
存于氨分子中, 利用氨的易储运特性, 在终端用户处通 过氨分解制氢、氨燃烧、氨燃料电池等方式释放能量, 即 是氨作为能源载体的含义 ${ }^{[3]}$.

目前工业合成氨采用的是 Haber-Bosch (H-B)工艺, 它需要在高温高压条件 $\left(350 \sim 550{ }^{\circ} \mathrm{C}\right.$, 压力 $10 \sim 30$ $\mathrm{MPa}$ )下实施，消耗全球能源供应总量的 $1 \% \sim 2 \%{ }^{[6]}$; 同 时, 该过程需要使用大量化石资源用于产氢和供能, 每 年排放大约 5 亿吨二氧化碳, 约占全球二氧化碳排放的 $1.8 \%$. 在节能减排和可持续发展为首要任务的今天, 开 发清洁高效的 “绿色” 合成氨工艺尤为重要和迫切 ${ }^{[7]}$.

目前, 由可再生能源驱动, 并通过多相催化、电催 化、光催化和化学链等方式进行的 “绿色” 合成氨的路 线大致有两条 ${ }^{[8]}$. 一是利用可再生能源制取氢气, 氢气 再和氮气进行反应生成氨, 如图 1 路线 1 所示; 事实上, 在 20 世纪 60 年代以前, 挪威、埃及等国曾利用本国丰 富的水电, 制取氢气, 氢气再和氮气反应合成氨. 另一 途径则是直接以氮气和水反应生成氨, 如图 1 路线 2 所 示, 该路线可跳过制氢过程, 减少中间环节, 但目前效 率还较低, 离实际应用还有较长一段距离.

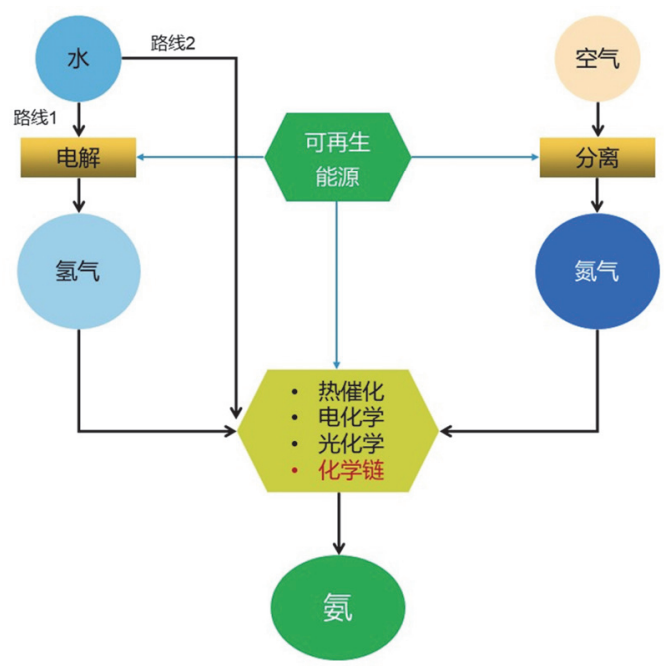

图 1 可再生能源驱动的 “绿色” 合成氨过程

Figure 1 Green ammonia synthesis processes driven by renewable energy

\section{2 化学链过程}

化学链过程(Chemical looping)是指将目标反应拆分 为两个或多个分步反应，各分步反应可在不同的空间、 时间或反应条件下分别进行, 分步反应可以逐一优化以 达到整个目标反应的最优化. 在化学链过程中, 常常需 要借助某种 “反应载体(Carrier)” 的消耗和再生完成整 个化学循环. 根据目标反应的不同, 反应载体可用于 氧、氮、碳或氢等物种的传递, 分别称之为载氧体 ${ }^{[9]}$ 、 载氮体 ${ }^{[10]}$ 、载碳体 ${ }^{[11]}$ 或载氢体 ${ }^{[12]}$ 等.

化学链过程与多相催化过程有着密切的关联. 二者 本质上均涉及气固或液固表界面化学反应, 有些多相催
化剂亦可用作化学链过程中的反应载体. 但是, 化学链 不同于多相催化过程，载体亦不同于多相催化剂. 二者 之间存在诸多不同, 现以催化与化学链过程合成氨反应 $\left(\mathrm{N}_{2}+\mathrm{H}_{2} \rightarrow \mathrm{NH}_{3}\right)$ 为例来具体说明二者之间的差别, 如图 2 所示.

反应物进料模式上, 多相催化过程一般采用共进料 模式; 而化学链过程则采取分步进料模式. 催化反应过 程中温度和压力需要保持恒定; 而化学链过程中, 各分 立步骤中的温度和压力可以不同. 催化过程中，反应物 及产物的浓度、催化剂的组成与结构、催化剂表面吸附 物种丰度等都基本保持不变; 而化学链是一非稳态过 程，反应物及产物的浓度、“反应载体” 的组成与结构等 在每一步反应过程中都会随时间的变化而变化. 因此, 与催化过程相比, 化学链过程具有一些独特的特点.
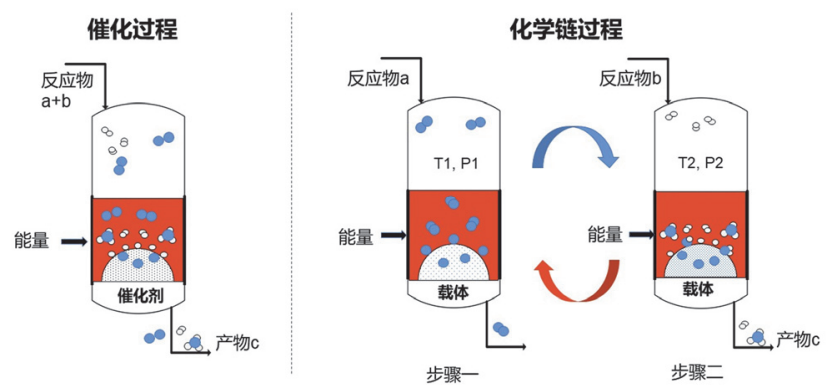

图 2 催化(a)与化学链(b)过程之对比

Figure 2 Comparison of catalytic process (a) with chemical looping process (b)

目前, 化学链技术已在化石能源燃烧与二氧化碳捕 获、水分解制氢、化学品及液体燃料制备等重要领域得 到了广泛的关注与研究. 如化学链燃烧(Chemical looping combustion, CLC) 具有 $\mathrm{CO}_{2}$ 内分离、能量利用率高、 能耗低、污染低等优点, 是一种很有前景、经济环保、 效益高的燃烧方式. 迄今为止, 国内外研究者进行了大 量卓有成效的工作 ${ }^{[13]}$.

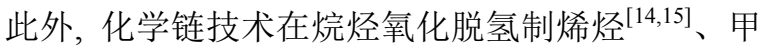
烷氧化制取甲醇 ${ }^{[16,17]}$ 、甲烷氧化偶联 ${ }^{[18]}$ 、甲烷制合成 气 ${ }^{[19,20]}$ 、水分解制氢 ${ }^{[21,22]}$ 、合成氨 ${ }^{[10]}$ 等领域也取得了许 多新的研究进展. 感兴趣的读者可参阅近期发表的相关 综述文章 ${ }^{[23,24]}$, 本综述仅详细介绍化学链合成氨的研究 进展.

\section{3 化学链合成氨}

\section{1 化学链合成氨的发展概况}

将合成氨催化过程解耦为固氮及产氨等两步或两 步以上分立的化学反应, 即为化学链合成氨过程. 早在 19 世纪时, Tessie du Motay 就曾提出利用 $\mathrm{Ti}_{3} \mathrm{~N}_{2}$ 和 $\mathrm{TiN}$ 之 间的循环转换合成氨 ${ }^{[25]}$, 即 $\mathrm{Ti}_{3} \mathrm{~N}_{2}$ 固氮生成富氮的 $\mathrm{TiN}$, $\mathrm{TiN}$ 与 $\mathrm{H}_{2}$ 高温反应放氨并再生贫氮的 $\mathrm{Ti}_{3} \mathrm{~N}_{2}$, 如方程式 (1), (2)所示. 
$2 \mathrm{Ti}_{3} \mathrm{~N}_{2}+\mathrm{N}_{2} \rightarrow 6 \mathrm{TiN}$

$6 \mathrm{TiN}+3 \mathrm{H}_{2} \rightarrow 2 \mathrm{Ti}_{3} \mathrm{~N}_{2}+2 \mathrm{NH}_{3}$

1908 年, Frank ${ }^{[26]}$ 开发了一种通过碳化钙 $\left(\mathrm{CaC}_{2}\right)$ 固氮 生成氰氨化钙 $\left(\mathrm{CaCN}_{2}\right)$, 继而氰氨化钙水解产氨的分步 合成氨方法, 水解产物碳酸钙与焦炭混合加热反应可再 生 $\mathrm{CaC}_{2}$, 其反应方程式如 (3) (6) 所示. 此方法在 Haber-Bosch 工艺开发前是工业上主要的固氮方法. 电 石再生的条件十分苛刻, 需要消耗大量的能量, 据估计 该工艺的能耗为 $210 \mathrm{GJ} \bullet \mathrm{t}^{-1} \mathrm{NH}_{3}$, 这使得该工艺在经济 上缺乏竞争力 ${ }^{[27]}$.

$\mathrm{CaC}_{2}+\mathrm{N}_{2} \rightarrow \mathrm{CaCN}_{2}+\mathrm{C} \quad \Delta H=-288.4 \mathrm{~kJ} \cdot \mathrm{mol}^{-1}$

$\mathrm{CaCN}_{2}+3 \mathrm{H}_{2} \mathrm{O} \rightarrow 2 \mathrm{NH}_{3}+\mathrm{CaCO}_{3} \quad \Delta H=-94.2 \mathrm{~kJ} \cdot \mathrm{mol}^{-1}$

$\mathrm{CaCO}_{3}+5 / 2 \mathrm{C} \rightarrow \mathrm{CaC}_{2}+3 / 2 \mathrm{CO}_{2} \quad \Delta H=557.8 \mathrm{~kJ} \cdot \mathrm{mol}^{-1}$

总包反应: $\mathrm{N}_{2}+3 \mathrm{H}_{2} \mathrm{O}+3 / 2 \mathrm{C} \rightarrow 2 \mathrm{NH}_{3}+3 / 2 \mathrm{CO}_{2}$

Haber 等 ${ }^{[28]}$ 曾经使用 $\mathrm{Mn}-\mathrm{Mn}_{3} \mathrm{~N}_{2}$ 和 $\mathrm{CaH}_{2}-\mathrm{Ca}_{3} \mathrm{~N}_{2}$ 进行 了化学链合成氨的尝试, 其反应方程式为 $(7) \sim(10)$. 但 该过程的合成氨产率极低, 因而未得到进一步研究.

$$
\begin{aligned}
& 3 \mathrm{CaH}_{2}+\mathrm{N}_{2} \rightarrow \mathrm{Ca}_{3} \mathrm{~N}_{2}+3 \mathrm{H}_{2} \\
& \mathrm{Ca}_{3} \mathrm{~N}_{2}+6 \mathrm{H}_{2} \rightarrow 3 \mathrm{CaH}_{2}+2 \mathrm{NH}_{3} \\
& 3 \mathrm{Mn}+\mathrm{N}_{2} \rightarrow \mathrm{Mn}_{3} \mathrm{~N}_{2} \\
& \mathrm{Mn}_{3} \mathrm{~N}_{2}+3 \mathrm{H}_{2} \rightarrow 3 \mathrm{Mn}+2 \mathrm{NH}_{3}
\end{aligned}
$$

自 20 世纪初 Haber-Bosch 合成氨工业建立以来, 关 于化学链合成氨的研究便逐渐淡出历史舞台. 近年来, 随着可再生能源合成氨的需求日益旺盛, 将太阳能、风 能等可再生能源与化学链过程相耦合, 使得化学链作为 一种替代合成氨方式得到了复兴 ${ }^{[29]}$. 与合成氨催化过 程相比, 化学链过程具有以下几个特点: 可在常压条件 下操作, 有助于简化工艺流程, 可用于分布化、小型化 产氨; 由于形成稳定的氮化物或氧化物, 易于启停, 易 于与可再生能源耦合; 可分别对固氮、产氨等各步骤中 的反应物、温度、压力等进行优化; 可规避 $\mathrm{N}_{2}$ 与 $\mathrm{H}_{2}$ 或 $\mathrm{H}_{2} \mathrm{O}$ 的竞争吸附问题.

根据载氮体(水解或加氢)放氨形式的不同, 化学链 合成氨过程可分为以水为氢源的化学链 (表示为 $\mathrm{H}_{2} \mathrm{O}-\mathrm{CL}$ ) 和以氢气为氢源的化学链过程 (表示为 $\mathrm{H}_{2}-\mathrm{CL}$ ), 如图 3 所示. 人们对于 $\mathrm{H}_{2} \mathrm{O}-\mathrm{CL}$ 过程的兴趣源于其避免 了昂贵的、高能耗的天然气或煤重整制氢过程, 是目前 受到关注较多的体系. 而对于 $\mathrm{H}_{2}-\mathrm{CL}$ 过程, 氢气则可来 源于可再生能源, 通过电解水等制氢方式得到, 再通过 化学链方式制氨.

在化学链合成氨过程中, 载氮体材料的选择是决定 过程效率的关键因素. 近年来, 随着化学链合成氨技术 逐渐引起学者的关注, 一系列载氮体得到了广泛而深入 的研究, 从而大大丰富了化学链合成氨的研究内涵. 接 下来, 就近期化学链合成氨载氮体材料的发展做一介 绍.

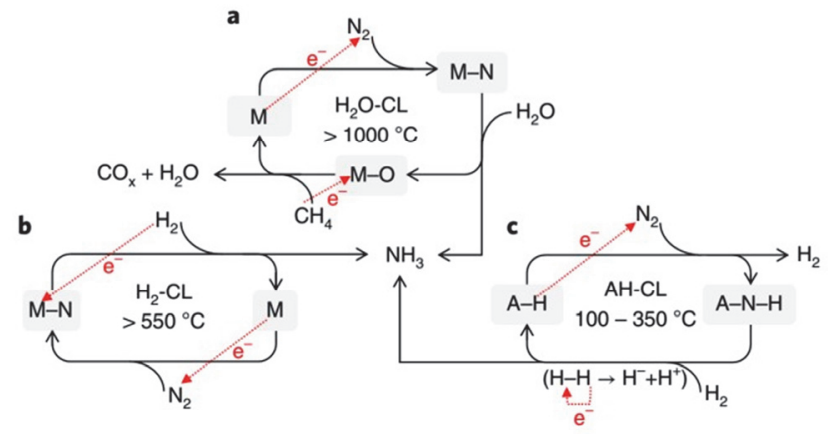

图 3 以水为氢源的 $\mathrm{H}_{2} \mathrm{O}-\mathrm{CL}$ 及以 $\mathrm{H}_{2}$ 为氢源的 $\mathrm{H}_{2}-\mathrm{CL}$ 和 AH-CL. $\mathrm{M}$ 为 $\mathrm{Al} 、 \mathrm{Cr} 、 \mathrm{Mn}$ 等金属, $\mathrm{A}$ 为碱(土)金属 ${ }^{[10]}$

Figure 3 Chemical looping processes for ammonia synthesis. M denotes metals such as $\mathrm{Al}, \mathrm{Cr}$ and $\mathrm{Mn}$; $\mathrm{A}$ denotes alkali or alkaline earth metal. Reprinted from Ref. [10] with permission from Nature Energy, Springer Nature

\section{2 载氮体材料的近期发展概况}

目前研究的载氮体材料大多是无机含氮化合物, 包 括金属氮化物(nitride)、氮氧化物(oxynitride)、氮氢化物 (nitride hydride)、(亚)氨基化合物(amide or imide)等，分 别构成氮化物-氧化物对、贫氮氮化物-富氮氮化物对或 氢化物-亚氨基化合物对等实现固氮及产氨反应. 根据 化学键性质的不同，氮化物一般可分为共价型氮化物、 过渡金属氮化物和离子型氮化物. 据此, 本综述将载氮 体材料分为以下三种.

\section{2 .1 共价型氮化物载氮体}

共价氮化物的主要代表是 AlN. 2007 年, Steinfeld 等 ${ }^{[30,31]}$ 设计了一种太阳能集热驱动的两步化学链合成 氨过程(图 4). 在固氮过程中, $\mathrm{Al}_{2} \mathrm{O}_{3}$ 在氮气气氛中与甲 烷或者碳等还原性原料共同反应生成 $\mathrm{AlN}$ 和 $\mathrm{CO} ; \mathrm{AlN}$ 与水发生高温水解反应，可以生成氨同时 AlN 重新回到 $\mathrm{Al}_{2} \mathrm{O}_{3}$. 其反应方程式及反应焓变如 $(11) \sim(13)$ 所示. 从 热力学上看, 第一步反应为强吸热过程, 反应需要高温 才能发生. 第二步则是放热反应.

$$
\begin{gathered}
\text { 固氮: } \mathrm{Al}_{2} \mathrm{O}_{3}+3 \mathrm{C}+\mathrm{N}_{2} \rightarrow 2 \mathrm{AlN}+3 \mathrm{CO} \\
\Delta H=708.1 \mathrm{~kJ} \cdot \mathrm{mol}^{-1} \\
\mathrm{Al}_{2} \mathrm{O}_{3}+3 \mathrm{CH}_{4}+\mathrm{N}_{2} \rightarrow 2 \mathrm{AlN}+6 \mathrm{H}_{2}+3 \mathrm{CO} \\
\Delta H=931.9 \mathrm{~kJ} \cdot \mathrm{mol}^{-1} \\
\text { 产氨: } 2 \mathrm{AlN}+3 \mathrm{H}_{2} \mathrm{O} \rightarrow 2 \mathrm{Al}_{2} \mathrm{O}_{3}+2 \mathrm{NH}_{3} \\
\Delta H=-274.1 \mathrm{~kJ} \cdot \mathrm{mol}^{-1}
\end{gathered}
$$

这一 $\mathrm{AlN}-\mathrm{Al}_{2} \mathrm{O}_{3}$ 化学链过程的特点在于原料 $\mathrm{Al}_{2} \mathrm{O}_{3}$ 的价格低廉、常压操作工艺、利用太阳能集热器供能、 无 $\mathrm{CO}_{2}$ 排放、副产 $\mathrm{CO}$ 等; 缺点为两步反应都需要高温 条件, 第一步需要 $1500 \sim 1700{ }^{\circ} \mathrm{C}$, 第二步需要 $900 \sim$ $1200{ }^{\circ} \mathrm{C}$, 能耗较大, 对反应器设备的要求较高. 且高温 时, 氨分解为氮气和氢气的反应是热力学自发进行的, 从而降低了氨的产率. 


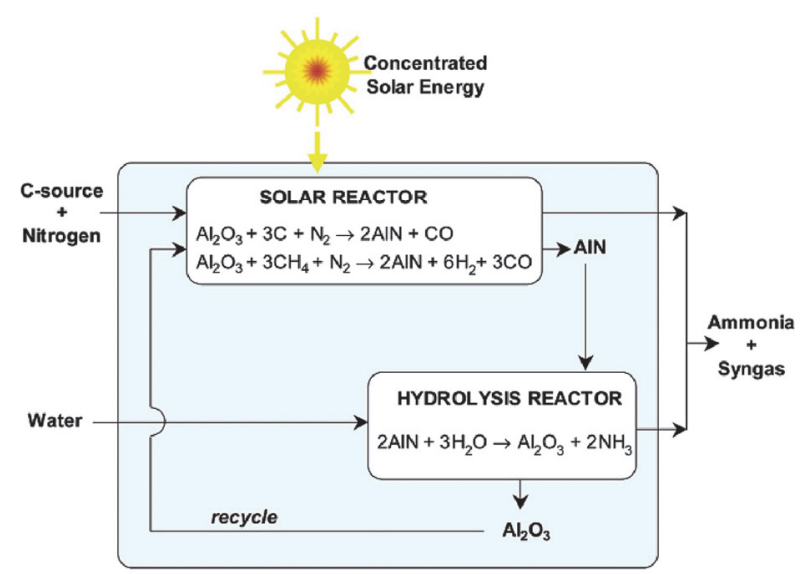

图 4 太阳能聚热驱动的 $\mathrm{AlN}-\mathrm{Al}_{2} \mathrm{O}_{3}$ 化学链合成氨工艺 ${ }^{[30]}$

Figure 4 An $\mathrm{AlN}-\mathrm{Al}_{2} \mathrm{O}_{3}$ CLAS process driven by solar energy. Reprinted from Ref. [30] with permission from Industrial \& Engineering Chemistry Research, American Chemical Society

研究者对于 $\mathrm{AlN}^{-} \mathrm{Al}_{2} \mathrm{O}_{3}$ 化学链合成氨过程的影响因 素, 如 $\mathrm{Al}_{2} \mathrm{O}_{3}$ 结构、催化剂、反应条件等进行了研究. $\mathrm{Al}_{2} \mathrm{O}_{3}$ 的结构和存在形式影响固氮反应速率, 反应性的 顺序为 $\gamma-\mathrm{Al}_{2} \mathrm{O}_{3}>\mathrm{Al}(\mathrm{OH})_{3}>\alpha-\mathrm{Al}_{2} \mathrm{O}_{3}{ }^{[32]}$. 为改善反应动力 学性能, 研究者尝试了多种添加剂或催化剂. 针对固氮 反应，研究发现一些含钙化合物，如 $\mathrm{CaF}_{2} 、 \mathrm{CaCO}_{3}$ 、 $\mathrm{Ca}(\mathrm{OH})_{2}$ 和 $\mathrm{CaC}_{2}$ 等在 $1350{ }^{\circ} \mathrm{C}$ 下与 $\mathrm{Al}_{2} \mathrm{O}_{3}$ 形成铝酸盐, 而铝酸盐在高温下气化离解形成铝蒸气, 可以快速氮 化. 此外, $\mathrm{Y}_{2} \mathrm{O}_{3} 、 \mathrm{Yb}_{2} \mathrm{O}_{3} 、 \mathrm{Cr}_{2} \mathrm{O}_{3} 、 \mathrm{Mg} 、 \mathrm{Na}_{2} \mathrm{CO}_{3} 、 \mathrm{Li}_{2} \mathrm{CO}_{3}$ 、 $\mathrm{NiCO}_{3}$ 等添加剂也可以提高反应(11)中原料的转化率 ${ }^{[32]}$.

对于水解产氨步骤， $\mathrm{Wu}$ 等 ${ }^{[33]}$ 发现 $\mathrm{Fe}_{2} \mathrm{O}_{3}$ 和 $\mathrm{CuO}$ 的 加入对 AlN 的水解反应有明显的催化作用, 其中 $\mathrm{Fe}_{2} \mathrm{O}_{3}$ 的催化作用优于 $\mathrm{CuO} . \mathrm{Fe}_{2} \mathrm{O}_{3}$ 的加入, 使得 $\mathrm{AlN}$ 水解反应 的表观活化能从 $186.3 \mathrm{~kJ} \cdot \mathrm{mol}^{-1}$ 降低到 $119.2 \mathrm{~kJ} \cdot \mathrm{mol}^{-1}$,

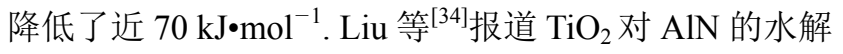
产氨反应亦表现出良好的催化作用, 这是由于 $\mathrm{TiO}_{2}$ 表 面具有良好的 $\mathrm{H}_{2} \mathrm{O}$ 解离吸附性能, 所产生的羟基在水解 反应中起着重要作用. 随着 $\mathrm{TiO}_{2}$ 的负载量、反应温度和 水蒸汽浓度的增加, $\mathrm{AlN}$ 的转化率和 $\mathrm{NH}_{3}$ 的产率都有所 增加. 近期, 他们 ${ }^{[35]}$ 又发现 $\mathrm{ZrO}_{2}$ 能促进 $\mathrm{NH}_{3}$ 的形成, 因 为 $\mathrm{NH}_{3}$ 分子吸附在 $\mathrm{ZrO}_{2}$ 表面, 从而阻止 $\mathrm{NH}_{3}$ 的分解. 温 度和水蒸气浓度的升高促进了 $\mathrm{AlN}$ 的水解和氨的生成, 但随着温度的升高, 氨的生成效率明显降低. 另外, 添 加过量的 $\gamma-\mathrm{Al}_{2} \mathrm{O}_{3}$ 可以增加 AIN 载氮体的孔隙率, 提高 固氮效率, 但是随着温度的升高, 载氮体的孔隙率明显 下降, 抑制放氨过程 ${ }^{[36]}$. 对于水解产氨反应, 如何抑制 高温下氨的分解, 是提高氨收率必须要考虑的因素.

\subsection{2 过渡金属氮化物载氮体}

与 $\mathrm{AlN}-\mathrm{Al}_{2} \mathrm{O}_{3}$ 不同, 过渡金属元素具有丰富的可变 化学价态, 可形成种类繁多的氮化物/氧化物, 且氮/氧 化物的稳定性较弱, 是目前受到关注较多的载氮体材 料. 尤其是 $\mathrm{Cr} 、 \mathrm{Mo} 、 \mathrm{Mn}$ 等载氮体材料得到了较为系统 的研究. 除了 $\mathrm{H}_{2} \mathrm{O}$ 直接作为反应物实施水解产氨反应
外，过渡金属氮化物还可以与 $\mathrm{H}_{2}$ 反应构成化学链合成 氨过程. 下面分别进行介绍.

\section{(1) $\mathrm{H}_{2} \mathrm{O}-\mathrm{CL}$}

2011 年, Michalsky 等 ${ }^{[37]}$ 提出了氮化铬作为载氮体, 利用 $\mathrm{Cr}-\mathrm{Cr}_{2} \mathrm{~N}-\mathrm{Cr}_{2} \mathrm{O}_{3}$ 间的循环, 通过 3 步化学反应合成氨 的化学链过程. 其反应方程式如(14) (17)所示. 首先, $\mathrm{Cr}_{2} \mathrm{O}_{3}$ 被 $\mathrm{CO}$ 或者 $\mathrm{H}_{2}$ 还原生成 $\mathrm{Cr}$, 第二步 $\mathrm{Cr}$ 被氮化生 成 $\mathrm{Cr}_{2} \mathrm{~N}$, 最后 $\mathrm{Cr}_{2} \mathrm{~N}$ 水解放氨.

$$
\begin{gathered}
\mathrm{Cr}_{2} \mathrm{O}_{3}+3 \mathrm{H}_{2} \rightarrow 2 \mathrm{Cr}+3 \mathrm{H}_{2} \mathrm{O} \quad \Delta H=360.0 \mathrm{~kJ} \cdot \mathrm{mol}^{-1} \\
\mathrm{Cr}_{2} \mathrm{O}_{3}+3 \mathrm{CO} \rightarrow 2 \mathrm{Cr}+3 \mathrm{CO}_{2} \quad \Delta H=278.6 \mathrm{~kJ} \cdot \mathrm{mol}^{-1} \\
2 \mathrm{Cr}+1 / 2 \mathrm{~N}_{2} \rightarrow \mathrm{Cr}_{2} \mathrm{~N} \quad \Delta H=-122.6 \mathrm{~kJ} \cdot \mathrm{mol}^{-1} \\
\mathrm{Cr}_{2} \mathrm{~N}+3 \mathrm{H}_{2} \mathrm{O} \rightarrow \mathrm{Cr}_{2} \mathrm{O}_{3}+\mathrm{NH}_{3}+3 / 2 \mathrm{H}_{2} \\
\Delta H=-321.1 \mathrm{~kJ} \cdot \mathrm{mol}^{-1}
\end{gathered}
$$

氧化铬的还原为强吸热反应, 从热力学上看, 需要 较高的温度. 在实验中, 由于避免了使用固态还原剂, 因而还原温度较 $\mathrm{AlN}^{-} \mathrm{Al}_{2} \mathrm{O}_{3}$ 体系有所降低, 但仍需要 $1200 \sim 1600{ }^{\circ} \mathrm{C}$ 的高温条件, 在 $1600{ }^{\circ} \mathrm{C}, \mathrm{Cr}$ 还原的速率 为 $2.7 \times 10^{-3} \mathrm{~mol}_{\mathrm{Cr}} \cdot \mathrm{mol}_{\mathrm{Cr} 2 \mathrm{O} 3}{ }^{-1} \cdot \mathrm{min}^{-1}$; 第二步和第三步是 放热反应, 但是由于存在动力学阻力, 实际反应中需要 $500 \sim 1000{ }^{\circ} \mathrm{C}$, 在 $1000{ }^{\circ} \mathrm{C}, \mathrm{Cr}$ 氮化的速度为 $4.13 \times$ $10^{-2} \mathrm{~mol}_{\mathrm{N} 2} \cdot \mathrm{mol}_{\mathrm{Cr}}{ }^{-1} \cdot \mathrm{min}^{-1}$, 首先生成 $\mathrm{Cr}_{2} \mathrm{~N}$, 继续氮化生 成 $\mathrm{CrN}$, 氮化铬水解生成氨的速率为 $1.07 \times 10^{-4} \mathrm{~mol}_{\mathrm{NH} 3}$. $\mathrm{mol}_{\mathrm{Cr}}{ }^{-1} \cdot \min ^{-1}$.

该工艺除了使用 $\mathrm{Cr}$ 基化合物作为载氮体, 还可以 将其他过渡金属设计成类似的循环，更一般的反应方程 式如(18) (20)所示 ${ }^{[27]}$. Michalsky 等 ${ }^{[38]}$ 对不同氮化物中 晶格氮的扩散动力学对合成氨性能的影响进行了详细 研究, 发现氮化物的离子性(Ionicity)与晶格氮的扩散系 数有重要的关联, 即晶格氮的扩散系数随氮化物离子性 的增加而增大(增大的离子性可增加氮化物中活性晶格 氮的体积浓度), 从而促进氮化物水解产氨. 这一认识 有助于指导研究人员通过理性的材料设计寻找更为合 适的化学链载氮体材料. 其中 $\mathrm{Mn}$ 基和 $\mathrm{Mo}$ 基氮化物被 认为是潜在的载氮体而得到了较多的研究. Michalsky 等对 $\mathrm{Mo}_{2} \mathrm{~N}-\mathrm{MoO}_{2}$ 化学链合成氨的过程进行了热力学和 经济分析, 结果表明, 当氨的产量为 $900 \mathrm{t} \cdot \mathrm{day}^{-1}$, 每吨 氨的成本为 $534 \pm 28$ 美元时, 经济上将具有可行性 ${ }^{[39]}$.

$$
\begin{aligned}
& a / b c \mathrm{M}_{c} \mathrm{O}_{d}+a d / b c \mathrm{R} \rightarrow a / b \mathrm{M}+a d / b c \mathrm{RO} \\
& a / b \mathrm{M}+1 / 2 \mathrm{~N}_{2} \rightarrow 1 / b \mathrm{M}_{a} \mathrm{~N}_{b}
\end{aligned}
$$

$1 / b \mathrm{M}_{a} \mathrm{~N}_{b}+a d / b c \mathrm{H}_{2} \mathrm{O} \rightarrow a / b c \mathrm{M}_{c} \mathrm{O}_{d}+\mathrm{NH}_{3}+(a d / b c-3 / 2) \mathrm{H}_{2}$

2017 年, Pfromm 等 ${ }^{[00]}$ 报道了一种用氮气、水蒸汽 和 $\mathrm{CH}_{4}$ 在大气压下制备 $\mathrm{NH}_{3}$ 和合成气 $\left(\mathrm{CO}-\mathrm{H}_{2}\right)$ 的新型化 学链过程: 首先, 金属锰与氮气反应生成 $\mathrm{Mn}_{5} \mathrm{~N}_{2}$, 接着 氮化锰在 $500{ }^{\circ} \mathrm{C}$ 水解生成 $\mathrm{NH}_{3}$ 和 $\mathrm{MnO}$, 第三步是 $\mathrm{MnO}$ 在 $1150{ }^{\circ} \mathrm{C}, 4 \% \mathrm{CH}_{4}-96 \% \mathrm{~N}_{2}$ 混合蒸汽中还原, 并再生 $\mathrm{Mn}_{5} \mathrm{~N}_{2}$, 构成化学链循环. 研究还发现, 在氮化物中加 入 $\mathrm{NaOH}$ 后，氨的产量有显著的提升，其中， $\mathrm{Na}$ 被认为 
是电子助剂, 削弱了 $\mathrm{Mn}-\mathrm{N}$ 键, 产生更多活性氮变成氨 气.

\section{(2) $\mathrm{H}_{2}-\mathrm{CL}$}

过渡金属氮化物通过加氢产氨的化学链产氨过程 可以表示为:

$$
\begin{aligned}
& \mathrm{TM}_{a} \mathrm{~N}_{b}+3 x / 2 \mathrm{H}_{2} \rightarrow \mathrm{TM}_{a} \mathrm{~N}_{b-x}+x \mathrm{NH}_{3} \\
& \mathrm{TM}_{a} \mathrm{~N}_{b-x}+x / 2 \mathrm{~N}_{2} \rightarrow \mathrm{TM}_{a} \mathrm{~N}_{b}
\end{aligned}
$$

在此过程中, 氮化物通过过渡金属的变价特性, 富 氮的氮化物与贫氮氮化物物相之间的转变实现产氨过 程. 过渡金属氮化物的稳定性是制约其固氮及加氢产氨 反应热力学和动力学性能的关键因素. 在过渡金属催化 的合成氨反应中, 我们已认识到过渡金属的催化性能符 合 Sabatier 原理, 即 $\mathrm{N}$ 物种在过渡金属上的吸附能应具 有适中的吸附强度 ${ }^{[41]}$. 这一原则可推广于载氮体的篮 选，事实上，早期人们就发现合成氨催化活性与过渡金 属氮化物的生成焓之间存在着火山型曲线的关系 ${ }^{[42]}$.

Michalsky 等 ${ }^{[43]}$ 对部分过渡金属氮化物载氮体固氮 及加氢产氨的热力学进行了计算. 从图 5 中可以看出, $\mathrm{Mn}_{4} \mathrm{~N}$ 及 $\mathrm{Cr}_{2} \mathrm{~N}$ 氮化为富氮的氮化物 $\left(\mathrm{Mn}_{5} \mathrm{~N}_{2}\right.$ 及 $\left.\mathrm{CrN}\right)$ 均为 热力学有利的反应, 而富氮氮化物的加氢产氨反应则是 热力学爬坡的反应; 与此不同的是, $\mathrm{Fe}$ 的氮化(生成 $\mathrm{Fe}_{4} \mathrm{~N}$ ) 则为热力学爬坡反应, $\mathrm{Fe}_{4} \mathrm{~N}$ 的加氢产氨则是热力 学可行的反应. 氮化锰具有丰富的价态和氮化物结构, 且较为适中的热力学, 因而以氮化锰为载氮体的研究较 多.

但是氮化锰的动力学性能较差, 如 $\mathrm{Mn}_{6} \mathrm{~N}_{2.58}$ 在 $550{ }^{\circ} \mathrm{C} 、 0.1 \mathrm{MPa}$ 氢气流的条件下加氢, 最终产物为 $\mathrm{Mn}_{4} \mathrm{~N}$, 反应速率为 $55 \mu \mathrm{mol}_{\mathrm{NH} 3} \bullet \mathrm{g}^{-1} \cdot \mathrm{h}^{-1}$. Laassiri 等 ${ }^{[44]}$ 报 道 $\mathrm{Mn}_{3} \mathrm{~N}_{2}$ 反应性很低, 只有 $3.1 \%$ 的晶格氮可与氢反应 生成氨. 因此, 不少研究着眼于对氮化锰的修饰改性. 为了降低氮化锰的相对稳定性, 锰基载氮体的设计关键 就在于降低晶格氮的稳定性. 晶格氮空位的生成能可作 为一个描述子(Descriptor), 用来衡量晶格氮转化为氨的 难易程度. 通过掺杂或合金化等方式对氮化锰的组成进 行调变, 可改变其局部或整体的电子结构, 从而调变过 渡金属氮化物晶格氮的稳定性, 最终改变产氨性能. Michalsky 等 ${ }^{[45]}$ 计算了掺杂不同过渡金属对 $\zeta-\mathrm{Mn}_{2} \mathrm{~N}$ 相 晶格氮空位的生成自由能的影响, 发现 $3 \mathrm{~d}$ 过渡金属(从 $\mathrm{Sc}$ 到 $\mathrm{Zn}$ )的加入都可以降低晶格氮的稳定性. 掺杂过渡 金属的 $\mathrm{d}$ 电子占据数越大, 晶格氮空位的生成自由能越 低. 实验证实, 与未掺杂相比, $\mathrm{Fe}$ 掺杂的氮化锰 $\left(\mathrm{Fe}_{0.25} \mathrm{Mn}_{1.75} \mathrm{~N}\right)$ 可以降低晶格氮的稳定性, 增加氮空位的 浓度. 最近, Liu 等 ${ }^{[46,47]}$ 利用密度泛函理论(DFT)计算了 $\mathrm{Mn}_{4} \mathrm{~N}$ 在分子水平上的加氢过程. 金属-氮 $(\mathrm{N}-\mathrm{M}, \mathrm{M}=$ $\mathrm{Mn}, \mathrm{Fe}, \mathrm{Ni})$ 和金属-氢 $(\mathrm{H}-\mathrm{M})$ 键的强度都决定了整个加 氢过程的热力学. 计算结果证实了 $\mathrm{Fe}$ 和 $\mathrm{Ni}$ 掺杂在氮化 锰的表层和次表层可以干扰其局域电子结构, 从而影响 产氨活性. 基于 DFT 的动力学模拟进一步表明, $\mathrm{Mn}_{4} \mathrm{~N}$
亚层中掺杂低浓度的 $\mathrm{Fe}$ 或 $\mathrm{Ni}$ 可降低晶格氮的扩散势垒. 此外，这些杂原子掺杂物种，特别是 $\mathrm{Ni}$ ，由于具有良好 的氢吸附作用，表层和次表层的 $\mathrm{Ni}$ 都可以降低还原反 应活化能.

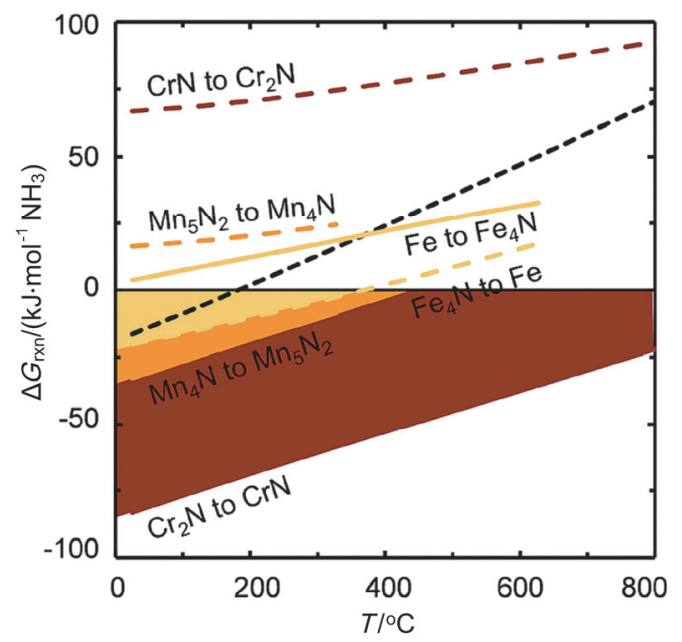

图 5 部分过渡金属及其氮化物进行固氮及加氢产氨反应的热力学计 算 ${ }^{[43]}$

Figure 5 Thermodynamic calculation of chemical looping ammonia synthesis by transition metals and their nitrides. Reprinted from Ref. [43] with permission from Chemical Science, Royal Society of Chemistry

金属 Co 的存在对氮化锰中氮转移亦会产生影响. 除过渡金属掺杂外，在氮化锰中掺杂低含量的 $\mathrm{Li}$, 可提 高低温下的合成氨性能. 在 Li-Mn-N 体系中, $400{ }^{\circ} \mathrm{C}$ 时, 有 $15 \%$ 的晶格氮与氢反应生成氨. 但是, $\mathrm{Li}-\mathrm{Mn}-\mathrm{N}$ 经氢 还原后具有较高的热化学稳定性, 导致在 $\mathrm{N}_{2}$ 气氛中的 再生步骤难以进行, 无法构成化学链过程 ${ }^{[44]}$.

Co-Mo-N 催化剂是继 $\mathrm{Fe} 、 \mathrm{Ru}$ 后发展的一类氮化物 合成氨催化剂 ${ }^{[48-50]}$. Hargreaves 等 ${ }^{[51-54]}$ 在研究 $\mathrm{Co}_{3} \mathrm{Mo}_{3} \mathrm{~N}$ 催化剂时, 发现 $\mathrm{H}_{2}$ 气氛中处理时, 部分晶格氮以氨的形 式释放出来，生成了贫氮的 $\mathrm{Co}_{6} \mathrm{Mo}_{6} \mathrm{~N}$ 相，而 $\mathrm{Co}_{6} \mathrm{Mo}_{6} \mathrm{~N}$ 相在 $\mathrm{N}_{2}$ 中焙烧后可再生 $\mathrm{Co}_{3} \mathrm{Mo}_{3} \mathrm{~N}$ 相，从而构成了合成 氨循环. $\mathrm{N}_{2}$ 同位素交换实验也证实晶格氮是活性氮，在 合成氨反应条件下直接参与了氨的催化合成 ${ }^{[53]}$. 而且, 对 $\mathrm{Co}_{6} \mathrm{Mo}_{6} \mathrm{~N}$ 进行实验时，发现除了存在氮交换，还可以 转变成 $\mathrm{Co}_{3} \mathrm{Mo}_{3} \mathrm{~N}$. 这项研究证实了 $\mathrm{Co}-\mathrm{Mo}-\mathrm{N}$ 体系中氮 的消耗和补充是可逆的，同时也表明 Co-Mo-N 也是一 种潜在的载氮体材料.

其它过渡金属氮化物作为载氮体的例子并不多. Hargreaves 等 ${ }^{[55,56]}$ 考察了一系列氮化物, 如 $\mathrm{Ni}_{3} \mathrm{~N} 、 \mathrm{Cu}_{3} \mathrm{~N}$ 、 $\mathrm{Zn}_{3} \mathrm{~N}_{2} 、 \mathrm{Ta}_{3} \mathrm{~N}_{5}$ 等在氢气气氛下的脱氮性能，其中 $\mathrm{Ni}_{3} \mathrm{~N}$ 和 $\mathrm{Cu}_{3} \mathrm{~N}$ 由于稳定性较低, 因此在相对较低温度 $\left(250{ }^{\circ} \mathrm{C}\right)$ 可以进行脱氮反应，脱氮率分别是 $25 \%$ 和 $30 \%$, 但是产物进行氨化再生氮化物却比较困难. 稳定性相对 较高的 $\mathrm{Zn}_{3} \mathrm{~N}_{2}$ 在 $400{ }^{\circ} \mathrm{C}$ 时有约 $23 \%$ 的氮转化成氨, 但是 产物氨化再生的量也很有限. 不同的是, $\mathrm{Ta}_{3} \mathrm{~N}_{5}$ 虽然传递 到氨的氮量很低 $(13 \%)$, 但是产物可以氨化再生. 氮化 
铁在 $400{ }^{\circ} \mathrm{C}$ 能脱掉 $70 \%$ 的氮，氮化钴在 $250{ }^{\circ} \mathrm{C}$ 可以完 全脱氮，氮化铼在 $350{ }^{\circ} \mathrm{C}$ 下也能脱去接近 $90 \%$ 的氮. 由 于这些氮化物的循环再生很困难, 难以形成化学链合成 氨过程, 但是这些研究对合成氨载氮体的设计与选择具 有借鉴意义.

\subsection{3 离子型氮化物载氮体}

$\mathrm{Li} 、 \mathrm{Mg} 、 \mathrm{Ca}$ 等与 $\mathrm{N}_{2}$ 生成相应的离子型氮化物 $\left(\mathrm{Li}_{3} \mathrm{~N} 、\right.$ $\mathrm{Mg}_{3} \mathrm{~N}_{2}$ 和 $\mathrm{Ca}_{3} \mathrm{~N}_{2}$ 等) 的反应不仅是热力学可行的, 且动力 学性能良好. 一个典型的例子是, 碱金属 $\mathrm{Li}$ 在室温就能 和 $\mathrm{N}_{2}$ 反应生成 $\mathrm{Li}_{3} \mathrm{~N}^{[57]}$. 这类离子型氮化物水解产氨的 速率也往往较快, 但由生成的氧化物或氢氧化物再生金 属或氮化物则是非常困难的. 比如 $\mathrm{MgO}$ 的碳热反应温 度高于 $1500{ }^{\circ} \mathrm{C}$. 此外, $\mathrm{LiOH}$ 的熔点较低 $\left(471{ }^{\circ} \mathrm{C}\right)$, 再生 $\mathrm{Li}_{3} \mathrm{~N}$ 时可能会因为熔化和气化从而带来产物分离的问 题.

2019 年, Halas 等 ${ }^{[58]}$ 提出了一种利用等离激元 (Plasmonic)材料 TiN 的光热转化的三步法合成氨过程: $\mathrm{TiN}$ 纳米颗粒在光照射作用下产生热, 驱动 $\mathrm{CH}_{4}$ 将 $\mathrm{MgO}$ 还原为金属 $\mathrm{Mg}$, 并生成物质的量比 $1: 2$ 的 $\mathrm{CO}$ 和 $\mathrm{H}_{2}$, 可以作为费托合成的原料. 第二步是金属镁在氮气气氛 中被氮化, 生成 $\mathrm{Mg}_{3} \mathrm{~N}_{2}$. 最后, $\mathrm{Mg}_{3} \mathrm{~N}_{2}$ 水解生成氨气, 再 生氧化镁, 完成循环. 由于材料只有有限的表面能够暴 露在光照下, 所以产氨速率很低, 约为 $1.67 \mu \mathrm{mol}_{\mathrm{NH} 3}$ • $\mathrm{g}_{\mathrm{TiN} / \mathrm{MgO}}{ }^{-1} \cdot \mathrm{h}^{-1}$. 为提高光热耦合化学链产氨速率, 需要 增大其光热转化效率或尝试其它等离激元材料.

$$
\begin{aligned}
& \mathrm{MgO}+\mathrm{CH}_{4} \rightarrow \mathrm{Mg}+\mathrm{CO}+2 \mathrm{H}_{2} \\
& 3 \mathrm{Mg}+\mathrm{N}_{2} \rightarrow \mathrm{Mg}_{3} \mathrm{~N}_{2} \\
& \mathrm{Mg}_{3} \mathrm{~N}_{2}+\mathrm{H}_{2} \mathrm{O} \rightarrow 3 \mathrm{MgO}+\mathrm{NH}_{3}
\end{aligned}
$$

除了光热耦合化学链合成氨外, 热电耦合是另一种 可再生能源利用的方式. 近期, Jaramillo 等 ${ }^{[59]}$ 提出了一 种基于 $\mathrm{Li}$ 循环的化学链过程, 该过程的化学反应如 (26) (28) 所示. 第一步是 $\mathrm{LiOH}$ 电解生成金属锂; 第二 步是将金属 $\mathrm{Li}$ 暴露在流动态的氮气中, 温度维持在 $22 \sim 100{ }^{\circ} \mathrm{C}$, 形成 $\mathrm{Li}_{3} \mathrm{~N}$; 第三步是 $\mathrm{Li}_{3} \mathrm{~N}$ 水解产生氨气. 作者通过电解的方式, 使该反应可以在 $300{ }^{\circ} \mathrm{C}$ 条件下 进行, 电流效率达到了 $88.5 \%$. 该过程中由 $\mathrm{LiOH}$ 生成 $\mathrm{Li}$ 的反应热力学不利, 电解过程需要消耗大量的电能. 该工作表明通过其他形式能量的注入, 可以调变反应的 热力学, 使反应在更温和条件下进行.

$$
\begin{aligned}
& 6 \mathrm{LiOH} \rightarrow 6 \mathrm{Li}+3 \mathrm{H}_{2} \mathrm{O}+3 / 2 \mathrm{O}_{2}(\mathrm{~g}) \\
& 6 \mathrm{Li}+\mathrm{N}_{2}(\mathrm{~g}) \rightarrow 2 \mathrm{Li}_{3} \mathrm{~N}(\mathrm{~s}) \\
& 2 \mathrm{Li}_{3} \mathrm{~N}(\mathrm{~s})+6 \mathrm{H}_{2} \mathrm{O} \rightarrow 6 \mathrm{LiOH}+2 \mathrm{NH}_{3}
\end{aligned}
$$

碱(土)金属氮化物作为载氮体，也可以利用加氢反 应产氨. Michalsky 等 ${ }^{[43]}$ 计算了碱(土)金属氮化物与氢化 物相互转化反应的热力学(图 6), 可以看出, 碱(土)金属 氢化物(Hydride)如 $\mathrm{LiH} 、 \mathrm{CaH}_{2}$ 和 $\mathrm{SrH}_{2}$ 转化成 $\mathrm{Li}_{3} \mathrm{~N} 、 \mathrm{Ca}_{3} \mathrm{~N}_{2}$ 和 $\mathrm{Sr}_{3} \mathrm{~N}_{2}$ 的反应高温有利, 碱(土)金属氮化物生成氢化 物则是低温有利的反应; 选择合适的温度及压力有可能
实现化学链合成氨. 作者考察了 $\mathrm{Ca}_{3} \mathrm{~N}_{2}$ 和 $\mathrm{Sr}_{2} \mathrm{~N}$ 与 $\mathrm{H}_{2}$ 的 反应，在 $550{ }^{\circ} \mathrm{C} 、 0.1 \mathrm{MPa}$ 条件下，二者加氢后的最终 产物分别为 $\mathrm{Ca}_{2} \mathrm{NH}$ 和 $\mathrm{SrH}_{2}$, 产氨速率分别为 98 和 81 $\mu \mathrm{mol}_{\mathrm{NH} 3} \cdot \mathrm{g}^{-1} \cdot \mathbf{h}^{-1}$. 但对于固氮反应, 作者并未进行考察.

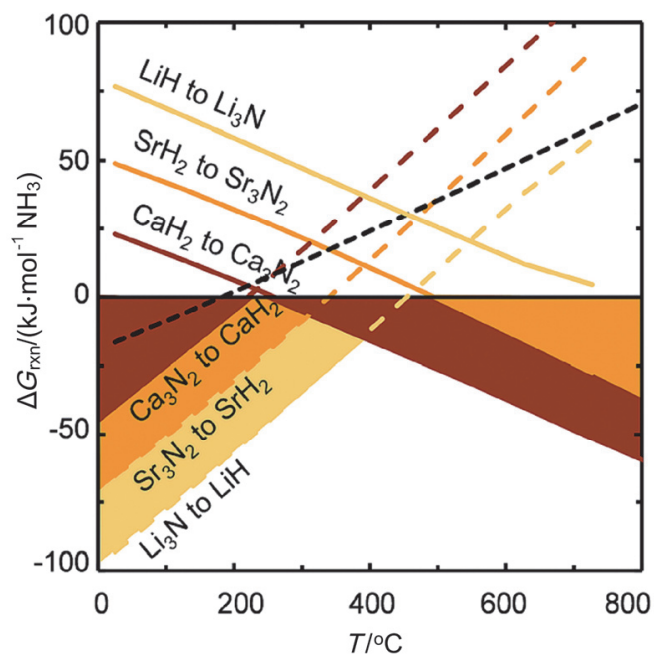

图 6 碱(土)金属氮化物及氢化物进行化学链合成氨的热力学计算 ${ }^{[43]}$ Figure 6 Thermodynamic calculation of chemical looping ammonia synthesis by alkali (earth) metal nitrides and their hydrides. Reprinted from Ref. [43] with permission from Chemical Science, Royal Society of Chemistry

Ichikawa 等 ${ }^{[60}$ 报道 $\mathrm{Li}_{3} \mathrm{~N}$ 在 $300{ }^{\circ} \mathrm{C}$ 和 $0.5 \mathrm{MPa} \mathrm{H}_{2}$ 流 中可生成 $\mathrm{LiH}$ 和 $\mathrm{NH}_{3}$. 最近, 他们发现金属 $\mathrm{Li}$ 可与 14 族金属元素如 $\mathrm{Sn}$ 等形成 $\mathrm{Li}-\mathrm{Sn}$ 合金 $\left(\mathrm{Li}_{17} \mathrm{Sn}_{4}\right), \mathrm{Li}-\mathrm{Sn}$ 合金 在 $400{ }^{\circ} \mathrm{C}$ 可以和氮气反应生成纳米 $\mathrm{Li}_{3} \mathrm{~N}$ 和 $\mathrm{Li}_{7} \mathrm{Sn}_{2}$ 合金 (式 29), $\mathrm{Li}_{3} \mathrm{~N}$ 和 $\mathrm{H}_{2}$ 反应生成 $\mathrm{LiH}$ 和 $\mathrm{NH}_{3}, 350{ }^{\circ} \mathrm{C}$ 后再生 Li-Sn 合金(式 30) ${ }^{[61,62]}$.

$$
\begin{aligned}
& 2 \mathrm{Li}_{17} \mathrm{Sn}_{4}+\mathrm{N}_{2} \rightarrow 2 \mathrm{Li}_{3} \mathrm{~N}+4 \mathrm{Li}_{7} \mathrm{Sn}_{2} \\
& 2 \mathrm{Li}_{3} \mathrm{~N}+4 \mathrm{Li}_{7} \mathrm{Sn}_{2}+3 \mathrm{H}_{2} \rightarrow 2 \mathrm{Li}_{17} \mathrm{Sn}_{4}+2 \mathrm{NH}_{3}
\end{aligned}
$$

近期, Gao 等 ${ }^{[10,63]}$ 提出了一种以碱(土)金属亚氨基 化合物为载氮体的低温化学链合成氨过程. 该过程利用 碱(土)金属氢化物与亚氨基化合物之间的相互转化实现 固氮及加氢产氨过程, 具体反应方程式如(31), (32)所 示. 在固氮步骤, 碱(土)金属氢化物与 $\mathrm{N}_{2}$ 反应生成相应 的亚氨基化合物，随后亚氨基化合物再与 $\mathrm{H}_{2}$ 反应放氨， 同时再生氢化物. 图 7 示出了 $0 \sim 450{ }^{\circ} \mathrm{C}$ 温度区间内, 部分二元氢化物, 如 $\mathrm{LiH} 、 \mathrm{MgH}_{2} 、 \mathrm{CaH}_{2} 、 \mathrm{BaH}_{2}$ 的固氮 及相应(亚)氨基化合物或氮化物, 如 $\mathrm{Li}_{2} \mathrm{NH} 、 \mathrm{CaNH}$ 、 $\mathrm{MgNH} 、 \mathrm{BaNH} 、 \mathrm{NaNH}_{2} 、 \mathrm{KNH}_{2}$ 及 $\mathrm{Mg}_{3} \mathrm{~N}_{2}$ 加氢产氨反应 的热力学. 可以看出, $\mathrm{LiH} 、 \mathrm{CaH}_{2} 、 \mathrm{MgH}_{2} 、 \mathrm{BaH}_{2}$ 等碱(土) 金属氢化物固氮生成相应亚氨基化合物或氮化物均是 热力学有利的反应, $\mathrm{NaH}$ 和 $\mathrm{KH}$ 由于难以形成稳定的亚 氨基化合物而无法实现固氮过程. 而亚氨基化合物或氮 化物加氢产氨则为热力学不利的反应, 如 $\mathrm{Mg}_{3} \mathrm{~N}_{2}$ 和 $\mathrm{MgNH}$, 在 $500{ }^{\circ} \mathrm{C}$ 以下难以实现加氢放氨过程. 氨基化 合物 $\mathrm{NaNH}_{2} 、 \mathrm{KNH}_{2}$ 在温度高于 $150{ }^{\circ} \mathrm{C}$ 时则是热力学可 行的反应. 综合考虑氢化物及亚氨基化合物的固氮及加 
氢产氨的热力学, $\mathrm{BaH}_{2}-\mathrm{BaNH}$ 和 $\mathrm{LiH}-\mathrm{Li}_{2} \mathrm{NH}$ 体系具有较 为合适的反应热力学, 有可能在较低温度和压力条件下 进行, 实施氨的化学链合成 ${ }^{[64]}$.

固氮:

$$
\mathrm{AH}_{x}+x \mathrm{~N}_{2} \rightarrow 2 x \mathrm{~A}_{2 / \mathrm{x}} \mathrm{NH}+x \mathrm{H}_{2}
$$

加氢放氨:

$x \mathrm{~A}_{2 / x} \mathrm{NH}+2 x \mathrm{H}_{2} \rightarrow 2 \mathrm{AH}_{x}+x \mathrm{NH}_{3}$

其中, $\mathrm{A}=\mathrm{Li} 、 \mathrm{Mg} 、 \mathrm{Ca} 、 \mathrm{Ba}$ 等; $x$ 是 $\mathrm{A}$ 的表观价态.

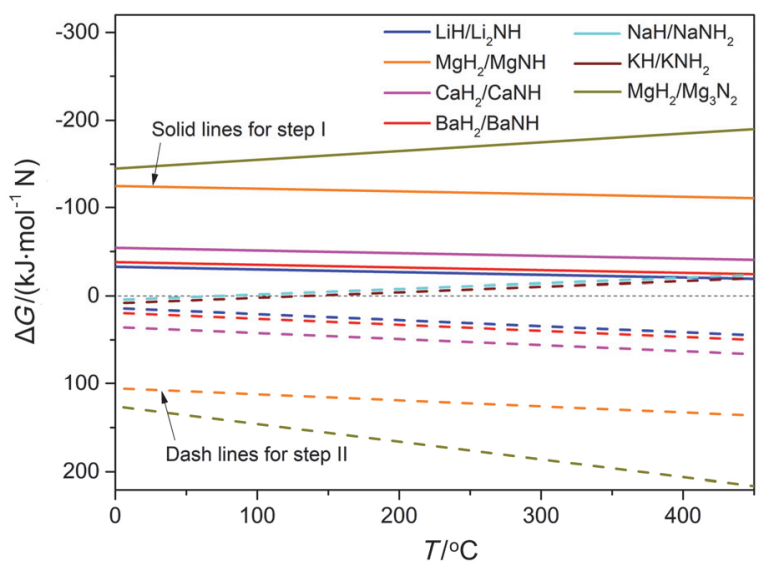

图 7 一系列二元碱(土)金属氢化物固氮和(亚)氨基化合物或氮化物 加氢产氨反应随温度变化的吉布斯自由能 ${ }^{[10]}$

Figure 7 Temperature-dependence Gibbs free energy of various alkali (earth) metal-N-H nitridation or hydrogenation. Reprinted from Ref. [10] with permission from Nature Energy, Springer Nature

实验结果证实了 $\mathrm{LiH}$ 和 $\mathrm{BaH}_{2}$ 分别在 400 和 $250{ }^{\circ} \mathrm{C}$ 即可实施固氮反应, 而 $\mathrm{Li}_{2} \mathrm{NH} 、 \mathrm{BaNH}$ 在 300 和 $200{ }^{\circ} \mathrm{C}$ 以上时可实现加氢产氨, 如图(8a 和 $8 b)$ 所示. 特别需要 指出的是, 氢化物的固氮过程是通过氢化物与 $\mathrm{N}_{2}$ 的氧 化还原反应完成的, 其中 $\mathrm{N}_{2}$ 被还原, 氢化物中的负氢物 种 $\left(\mathrm{H}^{-}\right)$失去电子生成质子和 $\mathrm{H}_{2}$. 这与过渡金属或碱 $($ 土 $)$ 金属作为电子给体还原 $\mathrm{N}_{2}$ 的反应是有显著区别的. 氢 化物的固氮及加氢产氨温度较 AIN 和过渡金属氮化物 均有大幅度降低. 研究还发现, $3 \mathrm{~d}$ 过渡金属如 Fe、Co 和 $\mathrm{Ni}$ 可作为催化剂显著加速氢化物固氮及亚氨基化合物 加氢产氨速率, 其催化效果顺序为 $\mathrm{Ni}>\mathrm{Co}>\mathrm{Fe}$. 在传统 的合成氨催化过程中, $\mathrm{Ni}$ 长期以来一直被认为是活性很 差的过渡金属 ${ }^{[65-67]}$, 这是由于 $\mathrm{N}_{2}$ 在金属 $\mathrm{Ni}$ 表面解离能 垒较高 ${ }^{[67]}$ 且 $\mathrm{H}_{2}$ 与 $\mathrm{N}_{2}$ 存在竞争吸附的缘故. 然而, $\mathrm{Ni}$ 加 速了氢化物的固氮反应速率这一事实表明了 $\mathrm{Ni}$ 与氢化 物之间存在着某种协同作用, 从而加速了 $\mathrm{N}_{2}$ 的解离. 研 究者在固定床反应器中, 通过交替通入 $\mathrm{N}_{2}$ 和 $\mathrm{H}_{2}$ 的操作 方式考察了氢化物-亚氨基化合物的化学链产氨速率. 如图 $8 \mathrm{c}$ 和 $8 \mathrm{~d}$ 所示, 与合成氨催化过程相比, 亚氨基化 合物作为载氮体的化学链产氨速率有了显著提高. 比如 $\mathrm{Al}_{2} \mathrm{O}_{3}$ 负载的 $\mathrm{Ni}-\mathrm{BaH}_{2}$ 在 $100{ }^{\circ} \mathrm{C}$ 时即实现了氨的合成 (2.3 $\mu \mathrm{mol} \cdot \mathrm{g}^{-1} \cdot \mathrm{h}^{-1}$ ), 在常压、 $250{ }^{\circ} \mathrm{C}$ 的产氨速率较催化
过程(使用高活性 $\mathrm{Cs}-\mathrm{Ru} / \mathrm{MgO}$ 为催化剂, $250{ }^{\circ} \mathrm{C}, 1 \mathrm{MPa}$ ) 高一个数量级(图 8d), 这一对比结果充分彰显了化学链 过程的优势. 研究者认为, 在化学链过程中 $\mathrm{N}_{2}$ 和 $\mathrm{H}_{2}$ 分 步进料的模式很大程度上避免了反应物在催化剂上的 竞争吸附，因而展现了明显的优势. 但是此过程中亚氨 基化合物的加氢放氨为吸热反应(图 7), 从热力学上看, 平衡氨浓度较低. 通过调变氢化物-亚氨基化合物的组 成可调变其热力学性能，从而提高产氨浓度. 此外，该 类材料的稳定性也是制约其实际应用的重要因素.

\subsection{4 其它载氮体}

2017 年, Steinfeld 等 ${ }^{[68]}$ 提出了一种由钻钛矿型氮氧 化物作为载氮体的化学链合成氨过程, 反应方程式如 (33)，(34)所示. 第一步，在高温和低氧分压下，利用太 阳能集热部分还原钙钛矿，产生氧空位用来活化和固定 氮气分子。第二步是氮氧化物水解产氨并再生钙钛矿氧 化物. 在这个过程中, 氧空位的产生被认为是速率控制 步骤. 钙钛矿化合物具有晶相稳定、组成可调等特点, 是燃料电池、二氧化碳和水的热化学裂解等研究领域受 到较多关注的材料, 其化学链合成氨性能还有待进一步 实验研究.

$$
\begin{aligned}
& \mathrm{ABO}_{3}+\varepsilon / 2 \mathrm{~N}_{2} \rightarrow \mathrm{ABO}_{3-\delta} \mathrm{N}_{\varepsilon}+\delta / 2 \mathrm{O}_{2} \\
& \mathrm{ABO}_{3-\delta} \mathrm{N}_{2 \delta / 3}+\delta \mathrm{H}_{2} \mathrm{O} \rightarrow \mathrm{ABO}_{3}+2 \delta / 3 \mathrm{NH}_{3}
\end{aligned}
$$

以上总结了近年来化学链合成氨的研究进展，表 1 对几种主要化学链合成氨载氮体的操作条件及产氨速 率进行了总结. 可以看到目前大部分载氮体材料的操作 温度较高, 产氨速率较低, 距实际应用还有较大的距离. 高效载氮体材料的开发是目前该领域所面临的主要挑 战.

\section{3 载氮体的选择及优化}

在化学链合成氨过程中, 载氮体通过固氮和加氢 (或水解)产氨的循环交替反应来实现合成氨循环. 载氮 体在此循环中既传递晶格氮，同时伴随着能量的传递过 程, 是整个化学链循环的核心. 为了实现高效化学链合 成氨过程，载氮体材料的开发是其关键核心技术。总体 而言，理想的载氮体应该具备以下几个条件：高的载氮 容量; 适中的固氮及加氢或水解产氨反应热力学; 良好 的动力学性能; 高的机械强度以及稳定性; 原料易得、 成本低; 制备能耗低且环境友好。

\subsection{1 载氮体的热力学性能优化}

化学链过程中, 载氮体参与的各分步反应的吉布斯 自由能变化 $(\Delta G)$ 是决定其性能优劣的关键因素. 因而, 选择载氮体材料时, 首先应对所有相关反应的热力学进 行分析 ${ }^{[27]}$. 理想的情形是，载氮体在一定的温度、压力 区间内，各反应均满足 $\Delta G \leqslant 0$ 的条件. 遗憾的是，这样 的载氮体目前尚未发现，如前文所述，载氮体材料的篮 选应符合 Sabatier 原理, 即载氮体中晶格氮的稳定性应 


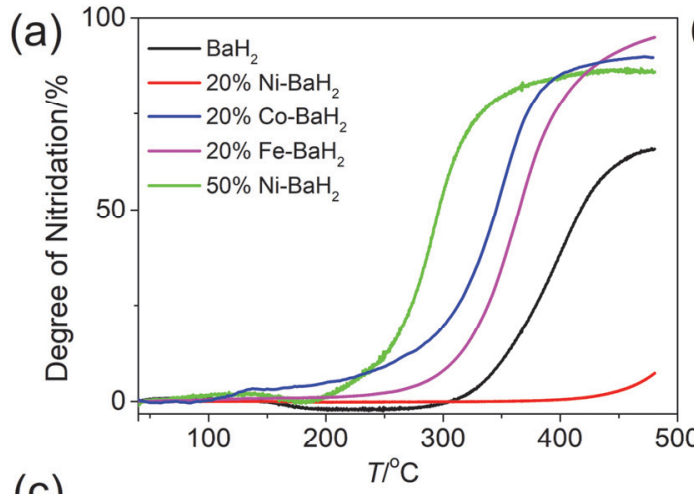

(c)
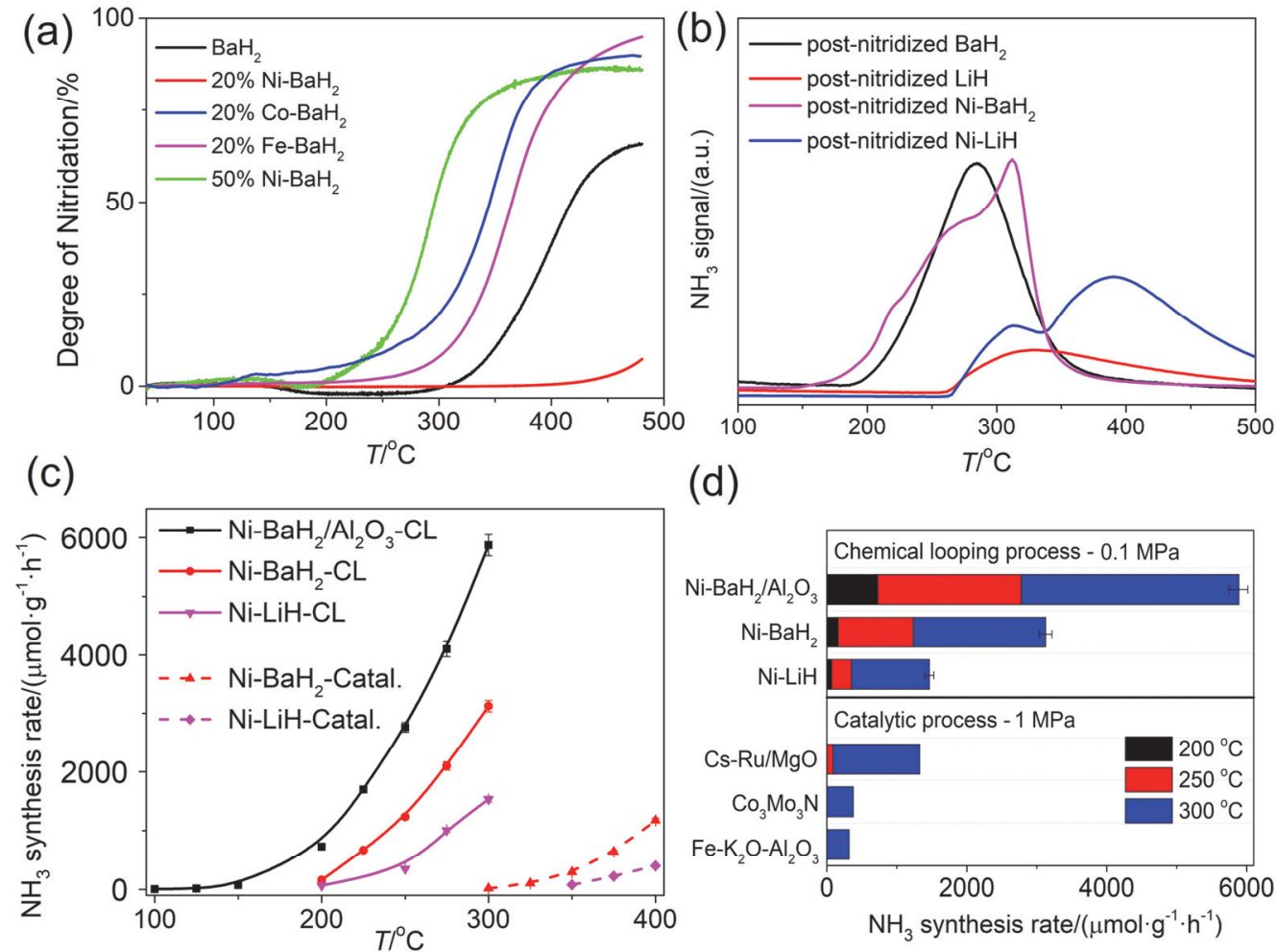

(d)

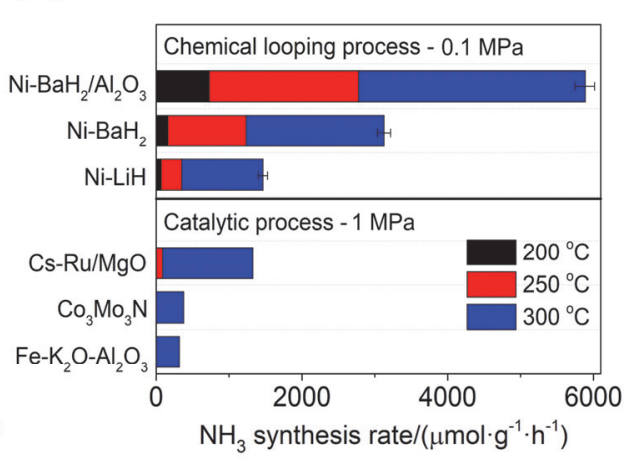

图 8 金属亚氨基化合物作为载氮体的化学链合成氨. (a) 氢化物的氮化过程. (b) 氮化后样品的加氢放氨. (c) Ni-氢化物化学链产氨速率与热催化 速率对比. (d) 氢化物化学链产氨速率与高活性合成氨催化剂热催化产氨速率的对比 ${ }^{[10]}$

Figure 8 Metal imides as nitrogen carriers for chemical looping ammonia synthesis. (a) Nitridation of hydride samples. (b) Hydrogenation of post-nitridized samples. (c) Temperature-dependent ammonia production rates of $\mathrm{Ni}-\mathrm{AH}$ samples in chemical looping and thermo-catalytic processes, respectively. (d) Comparison of $\mathrm{NH}_{3}$ production rates of Ni catalyzed AH-CL ( $\left.0.1 \mathrm{MPa}\right)$ and the conventional thermo-catalytic process $(1 \mathrm{MPa})$. Reprinted from Ref. [10] with permission from Nature Energy, Springer Nature

表 1 常见 CLAS 过程总结

Table 1 Summary of nitrogen carriers for CLAS

\begin{tabular}{|c|c|c|c|c|c|c|}
\hline No. & 载氮体对 & 反应 & 反应条件 & 产氨速率 $/\left(\mu \mathrm{mol} \cdot \mathrm{g}^{-1} \cdot \mathrm{h}^{-1}\right)$ & 特点 & Ref. \\
\hline 1 & $\mathrm{AlN}-\mathrm{Al}_{2} \mathrm{O}_{3}$ & $\begin{array}{l}\mathrm{Al}_{2} \mathrm{O}_{3}+3 \mathrm{C}+\mathrm{N}_{2} \rightarrow \\
2 \mathrm{AlN}+3 \mathrm{CO} \\
2 \mathrm{AlN}+3 \mathrm{H}_{2} \mathrm{O} \rightarrow \\
\mathrm{Al}_{2} \mathrm{O}_{3}+2 \mathrm{NH}_{3}\end{array}$ & $\begin{array}{l}\text { 固氮: } 0.1 \mathrm{MPa}, \\
1500 \sim 1700{ }^{\circ} \mathrm{C} \\
\text { 水解: } 0.1 \mathrm{MPa} \text {, } \\
900 \sim 1200{ }^{\circ} \mathrm{C}\end{array}$ & 无数据 & $\begin{array}{l}\text { 高温反应, 动力学性能差, 碳基 } \\
\text { 化合物作为还原剂, 太阳能聚 } \\
\text { 热 }\end{array}$ & {$[30,31]$} \\
\hline 2 & $\mathrm{Cr}_{2} \mathrm{~N}-\mathrm{Cr}_{2} \mathrm{O}_{3}$ & $\begin{array}{l}\mathrm{Cr}_{2} \mathrm{O}_{3}+3 \mathrm{H}_{2} \rightarrow \\
2 \mathrm{Cr}+3 \mathrm{H}_{2} \mathrm{O} \\
2 \mathrm{Cr}+1 / 2 \mathrm{~N}_{2} \rightarrow \mathrm{Cr}_{2} \mathrm{~N} \\
\mathrm{Cr}_{2} \mathrm{~N}+3 \mathrm{H}_{2} \mathrm{O} \rightarrow \\
\mathrm{Cr}_{2} \mathrm{O}_{3}+\mathrm{NH}_{3}+ \\
3 / 2 \mathrm{H}_{2}\end{array}$ & $\begin{array}{l}\text { 还原: } 0.1 \mathrm{MPa}, \\
1200 \sim 1500{ }^{\circ} \mathrm{C} \\
\text { 固氮: } 0.1 \mathrm{MPa} \text {, } \\
500 \sim 1000{ }^{\circ} \mathrm{C} \\
\text { 水解: } 0.1 \mathrm{MPa} \text {, } \\
500 \sim 1000{ }^{\circ} \mathrm{C}\end{array}$ & $\begin{array}{l}1000{ }^{\circ} \mathrm{C} \text { 时, } \mathrm{Cr}_{2} \mathrm{~N} \text { 水解产氨速 } \\
\text { 率为 } 108 \mu \mathrm{mol} \cdot \mathrm{g}^{-1} \cdot \mathrm{h}^{-1}\end{array}$ & $\begin{array}{l}\text { 高温反应, 动力学性能差, } \mathrm{H}_{2} \text { 为 } \\
\text { 还原剂, 过渡金属作为电子给 } \\
\text { 体还原 } \mathrm{N}_{2}\end{array}$ & [37] \\
\hline 3 & $\begin{array}{l}\mathrm{M}_{a} \mathrm{~N}_{b}-\mathrm{M}_{a} \mathrm{~N}_{b-d} \\
(\mathrm{Mn})\end{array}$ & $\begin{array}{l}\mathrm{M}_{a} \mathrm{~N}_{b-d}+d / 2 \mathrm{~N}_{2} \rightarrow \\
\mathrm{M}_{a} \mathrm{~N}_{b} \\
\mathrm{M}_{a} \mathrm{~N}_{b}+3 d / 2 \mathrm{H}_{2} \rightarrow \\
\mathrm{M}_{a} \mathrm{~N}_{b-d}+d \mathrm{NH}_{3}\end{array}$ & $\begin{array}{l}\text { 固氮: } 200 \sim \\
1000{ }^{\circ} \mathrm{C} \\
\text { 加氢: } 0.1 \mathrm{MPa}, \\
>300{ }^{\circ} \mathrm{C}\end{array}$ & $\begin{array}{l}\mathrm{Mn}_{6} \mathrm{~N}_{2.58} \text { 在 } 550{ }^{\circ} \mathrm{C} \text { 时加氢产 } \\
\text { 氨速率为 } 55.3 \mu \mathrm{mol} \cdot \mathrm{g}^{-1} \cdot \mathrm{h}^{-1}\end{array}$ & $\begin{array}{l}\text { 动力学性能差, 过渡金属作为 } \\
\text { 电子给体还原 } \mathrm{N}_{2}\end{array}$ & {$[43]$} \\
\hline 4 & $\begin{array}{l}\mathrm{MH}_{2}-\mathrm{M}_{\mathrm{a}} \mathrm{N}_{\mathrm{b}} \\
(\mathrm{Ca}, \mathrm{Sr})\end{array}$ & $\begin{array}{l}\mathrm{MH}_{2}+b / 2 \mathrm{~N}_{2} \rightarrow \\
\mathrm{M}_{a} \mathrm{~N}_{b}+a \mathrm{H}_{2} \\
\mathrm{M}_{a} \mathrm{~N}_{b}+(a+ \\
3 / 2 b) \mathrm{H}_{2} \rightarrow \\
a \mathrm{MH}_{2}+b \mathrm{NH}_{3}\end{array}$ & $\begin{array}{l}\text { 固氮: } 200 \sim \\
1000{ }^{\circ} \mathrm{C} \\
\text { 加氢: } 0.1 \mathrm{MPa}, \\
>300{ }^{\circ} \mathrm{C}\end{array}$ & $\begin{array}{l}\mathrm{Ca}_{3} \mathrm{~N}_{2} \text { 和 } \mathrm{Sr}_{2} \mathrm{~N} \text { 在 } 550{ }^{\circ} \mathrm{C} \text { 时的 } \\
\text { 加氢产氨速率分别为 } 98 \text { 和 } 81 \\
\mu \mathrm{mol} \cdot \mathrm{g}^{-1} \cdot \mathrm{h}^{-1}\end{array}$ & $\mathrm{H}^{-}$作为电子和质子给体还原 $\mathrm{N}_{2}$ & [43] \\
\hline 5 & $\mathrm{Mg}-\mathrm{Mg}_{3} \mathrm{~N}_{2}-\mathrm{MgO}$ & $\begin{array}{l}\mathrm{MgO}+\mathrm{CH}_{4} \rightarrow \\
\mathrm{Mg}+\mathrm{CO}+2 \mathrm{H}_{2} \\
3 \mathrm{Mg}+\mathrm{N}_{2} \rightarrow \mathrm{Mg}_{3} \mathrm{~N}_{2} \\
\mathrm{Mg}_{3} \mathrm{~N}_{2}+3 \mathrm{H}_{2} \mathrm{O} \rightarrow \\
3 \mathrm{MgO}+2 \mathrm{NH}_{3}\end{array}$ & $\begin{array}{l}\text { 光源加热 } \\
\text { 还原\&水解: } \\
\text { 压力 }<13.3 \mathrm{~Pa} \\
\text { 固氮: } 0.1 \mathrm{MPa}\end{array}$ & $1.67 \mu \mathrm{mol} \cdot \mathrm{g}^{-1} \cdot \mathrm{h}^{-1}$ & 光致等离子体加热 & [58] \\
\hline
\end{tabular}




\begin{tabular}{|c|c|c|c|c|c|c|}
\hline No. & 载氮体对 & 反应 & 反应条件 & 产氨速率 $/\left(\mu \mathrm{mol} \cdot \mathrm{g}^{-1} \cdot \mathrm{h}^{-1}\right)$ & 特点 & Ref. \\
\hline 6 & $\begin{array}{l}\mathrm{BaH}_{2}-\mathrm{BaNH} \\
\mathrm{LiH}-\mathrm{Li}_{2} \mathrm{NH}\end{array}$ & $\begin{array}{l}\text { 以 } \mathrm{Ba} \text { 为例: } \\
\mathrm{BaH}_{2}+\mathrm{N}_{2} \rightarrow \\
\mathrm{BaNH}+1 / 2 \mathrm{H}_{2} \\
\mathrm{BaNH}+2 \mathrm{H}_{2} \rightarrow \\
\mathrm{BaH}_{2}+\mathrm{NH}_{3} \\
\end{array}$ & $\begin{array}{l}\text { 固氮: } 0.1 \mathrm{MPa} \text {, } \\
100 \sim 500{ }^{\circ} \mathrm{C} \\
\text { 加氢: } 0.1 \mathrm{MPa}, \\
100 \sim 500{ }^{\circ} \mathrm{C} \\
\mathrm{Ni} \text { 催化剂 }\end{array}$ & $\begin{array}{l}300{ }^{\circ} \mathrm{C} \text { 时 } \mathrm{BaH}_{2}-\mathrm{BaNH} \\
\text { 的总包反应速率为 } \\
5870 \mu \mathrm{mol} \cdot \mathrm{g}^{-1} \cdot \mathrm{h}^{-1}\end{array}$ & $\begin{array}{l}\mathrm{H}^{-} \text {作为电子和质子给体还 } \\
\text { 原 } \mathrm{N}_{2}, \mathrm{H}_{2} \text { 异裂产生 } \mathrm{H}^{+} \text {和 } \mathrm{H}^{-}\end{array}$ & {$[10]$} \\
\hline 7 & $\mathrm{Li}-\mathrm{Li}_{3} \mathrm{~N}-\mathrm{LiOH}$ & $\begin{array}{l}6 \mathrm{LiOH} \rightarrow \\
6 \mathrm{Li}+3 \mathrm{H}_{2} \mathrm{O}+3 / 2 \mathrm{O}_{2} \\
6 \mathrm{Li}+\mathrm{N}_{2} \rightarrow 2 \mathrm{Li}_{3} \mathrm{~N} \\
2 \mathrm{Li}{ }_{3} \mathrm{~N}+6 \mathrm{H}_{2} \mathrm{O} \rightarrow \\
6 \mathrm{LiOH}+2 \mathrm{NH}_{3} \\
\end{array}$ & $\begin{array}{l}\text { 电解: } 0.1 \mathrm{MPa} \text {, } \\
450{ }^{\circ} \mathrm{C}, 3 \mathrm{~V} \\
\text { 固氮\&水解: } 0.1 \\
\mathrm{MPa}, 22 \sim 100{ }^{\circ} \mathrm{C}\end{array}$ & 无数据 & 电热耦合 & [59] \\
\hline 8 & $\mathrm{Mn}_{5} \mathrm{~N}_{2}-\mathrm{MnO}$ & $\begin{array}{l}\mathrm{Mn}_{5} \mathrm{~N}_{2}+5 \mathrm{H}_{2} \mathrm{O} \rightarrow \\
5 \mathrm{MnO}+2 \mathrm{NH}_{3}+2 \mathrm{H}_{2} \\
5 \mathrm{MnO}+5 \mathrm{CH}_{4}+\mathrm{N}_{2} \rightarrow \\
\mathrm{Mn}_{5} \mathrm{~N}_{2}+10 \mathrm{H}_{2}+5 \mathrm{CO} \\
\end{array}$ & $\begin{array}{l}\text { 水解: } 0.1 \mathrm{MPa} \text {, } \\
500{ }^{\circ} \mathrm{C} \\
\text { 固氮: } 0.1 \mathrm{MPa} \text {, } \\
1150{ }^{\circ} \mathrm{C}\end{array}$ & 无数据 & 过渡金属价态发生变化 & [40] \\
\hline
\end{tabular}

适中. 以此为理论依据, 热力学计算可高通量笁选载氮 体材料, 可大大缩小材料选择的范围, 为载氮体材料的 开发提供了强有力的工具.

Bartel 等 ${ }^{[69]}$ 对 1148 对二元金属氮化物-金属氧化物 构成的化学链合成氨过程进行了高通量的热力学计算. 对于包括氮化物水解产氨并生成氧化物、 $\mathrm{H}_{2}$ 还原氧化物 以及固氮再生金属氮化物的三步化学链产氨过程, 计算 结果揭示了循环中反应极限吉布斯自由能与氮化物和 氧化物的生成能之间呈现一火山型曲线(图 9), 其中 $\mathrm{Mn} 、 \mathrm{Fe} 、 \mathrm{~W} 、 \mathrm{Tc}$ 和 $\mathrm{Yb}$ 的氮化物-氧化物对可能是良好 的载氮体材料. 事实上, $\mathrm{Mn}$ 基氮化物正是目前研究较多 的载氮体材料之一。平衡产物分布分析表明除了 $\mathrm{CrN}-\mathrm{Cr}_{2} \mathrm{O}_{3} 、 \mathrm{MoN}-\mathrm{MoO}_{2} 、 \mathrm{WN}_{2}-\mathrm{WO}_{3} 、 \mathrm{MnN}-\mathrm{MnO}$ 和 $\mathrm{Mn}_{2} \mathrm{~N}-\mathrm{MnO}$ 外, $\mathrm{B} 、 \mathrm{~V} 、 \mathrm{Fe}$ 和 $\mathrm{Ce}$ 的氮化物是潜在的新型 载氮体, 值得进一步实验验证.

需要说明的是, 这项研究工作仅考察了二元氮化物 材料，但这些化合物只占已知材料的 $10 \%$. 因此，载氮 体材料开发的空间还很大. 一方面, 可以通过高通量热 力学计算快速篮选混合阳离子的三元或多元氮化物材 料. 在此过程中, 除了对已知的氮化物进行反应热力学
分析外，还需要人工设计新型的氮化物结构，从而帮助 实验研究人员缩小材料笁选范围，选择性的合成氮化物 材料并验证其固氮、产氨反应的动力学性能. 另一方面, 除了对氮化物的金属阳离子组成进行调变外，也可将 $\mathrm{O} 、 \mathrm{C} 、 \mathrm{H} 、 \mathrm{~F} 、 \mathrm{Cl} 、 \mathrm{Br}$ 等阴离子引入氮化物晶格, 形成 混合阴离子化合物, 如氮氧化物、氮碳化物、氮氢化物 等, 来调变氮化物的热力学性能. 相较于前者, 阴离子 调变更具有挑战性. 目前, 这类混合阴离子化合物还很 少，人们对于这类材料的合成、性质、反应等还知之甚 少. 仅有几个作为载氮体的例子, 因此, 混合阴离子载 氮体材料的设计与开发是一个非常值得探索的崭新领 域.

\subsection{2 载氮体的动力学性能优化}

对于具有良好热力学性能的载氮体材料, 考察其固 氮及产氨动力学性能是接下来需要做的工作. 由于化学 链合成氨过程为非平衡态过程, 涉及到表面反应、晶格 氮/氧/氢等物种在固相中的传递以及相变过程等, 动力 学阻力往往较大. 在固氮反应中, $\mathrm{N}_{2}$ 分子的解离吸附通 常被认为是动力学上的慢步骤. 在这种情况下, 可加入 合适的催化剂，比如过渡金属 $\mathrm{Ni}$ 的加入使得 $\mathrm{BaH}_{2}$ 固氮
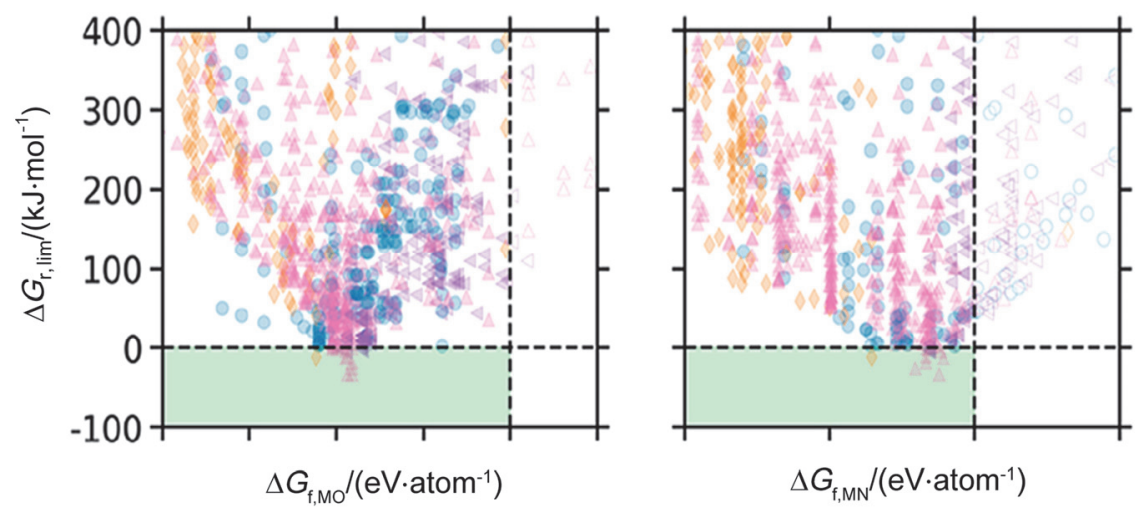

图 9 反应极限吉布斯自由能与氮化物和氧化物的生成能之间呈现一火山型曲线 ${ }^{[69]}$

Figure 9 Volcano dependence of solar thermochemical ammonia synthesis energetics. Reprinted from Ref. [69] with permission from ACS Applied Materials \& Interfaces, American Chemical Society 
反应的表观活化能从 $109 \mathrm{~kJ} \bullet \mathrm{mol}^{-1}$ 大幅降低至 $46 \mathrm{~kJ} \bullet$ $\mathrm{mol}^{-1}$, 从而加快了固氮速率 ${ }^{[10]}$. 在产氨反应中, 已有研 究表明, 对于过渡金属氮化物载氮体, 高温时的速控步 可能是固相扩散 ${ }^{[38]}$; 在这种情况下, 可通过掺杂、合金 化等方式削弱金属-氮键的强度, 降低晶格氮的稳定性.

总的来说，一种性能良好的载氮体不仅需要适中的 热力学性能, 更要结合动力学性能统一考量. 所以, 从 热力学和动力学两方面对载氮体的性能进行优化仍然 是化学链合成氨研究领域的重要课题.

\section{4 结论与展望}

可再生能源驱动的化学链合成氨过程是实现 “绿 色” 合成氨的重要途径之一. 在过去的十多年里, 研究 人员对化学链合成氨进行了积极的探索, 特别是对于不 同载氮体作为媒介的固氮及(水解或加氢)产氨反应的热 力学进行了大量的分析, 这对于载氮体材料的设计与开 发具有重要的指导意义. 尽管近年来化学链合成氨研究 取得了诸多进展, 总体来看, 产氨速率低仍是制约该过 程走向应用的首要障碍. 高性能载氮体材料的开发应是 近期化学链合成氨研究的核心内容.

对于 $\mathrm{H}_{2} \mathrm{O}-\mathrm{CL}$, 金属氧化物还原为金属或直接氮化 为氮化物的反应常常是热力学不利的, 且动力学阻力较 大, 需要高温的苛刻条件, 从而对反应器提出了很高的 要求. 开发三元或多元氧化物, 以降低二元金属氧化物 的热力学稳定性, 是值得研究和验证的可行策略之一. 相比于 $\mathrm{H}_{2} \mathrm{O}-\mathrm{CL}$ 过程, 过渡金属氮化物如氮化锰的固氮 和加氢产氨反应(即 $\mathrm{H}_{2}-\mathrm{CL}$ ) 的热力学较为适中, 是非常 具有前景的载氮体材料. 但是, 其动力学阻力较大, 产 氨速率低. 如何改善其固氮及加氢产氨的动力学性能是 目前研究的重点. 碱(土)金属亚氨基化合物, 作为一类 新型的载氮体材料, 具有适中的固氮及加氢产氨热力 学; 在过渡金属的催化作用下, 其展示出了良好的低温 动力学性能, 是目前文献报道的具有最高化学链产氨速 率的载氮体材料. 除对已有载氮体材料进行热力学和动 力学调变外, 新型载氮体的探索与开发仍将持续, 也是 这一领域长期的研究课题.

载氮体材料在化学链过程中的形貌、组成及结构变 化是影响其性能的重要因素, 但目前对于这方面的认识 还不够深入. 随着先进材料表征技术及理论计算的发 展, 将极大促进人们对于化学链过程微观机制的理解与 认识. 比如原位电镜、同步辐射表征手段等可以在原位 条件下观察载氮体的氮传递过程, 理论计算则可以从原 子水平上深入理解载氮体与 $\mathrm{N}_{2} 、 \mathrm{H}_{2}$ 的相互作用及催化 剂的作用机制等, 从而为载氮体材料的设计提供新的研 究思路.

除太阳能集热外, 将光、电、等离子体等不同能量 输入形式与化学链合成氨过程相耦合是目前该领域研 究的新动向. 光、电等外界能量的输入可以打破载氮体
的固氮及产氨反应的热力学平衡限制; 同时，对反应动 力学、中间物种的种类及浓度、反应路径等亦会产生不 同程度的影响. 考察这些影响因素与化学链产氨性能的 关联是该领域一个新的研究课题.

反应器的设计也是影响化学链过程效率的重要因 素. 化学链反应器包括固定床和流化床反应器等. 目前 化学链合成氨的研究主要集中在载氮体的设计与优化, 反应器大多以固定床反应器为主, 关于反应器的设计与 优化方面的研究还比较少, 有待进一步研究. 此外, 化 学链合成氨过程的生命周期分析、能量效率、成本核算 等也应随着载氮体材料的开发逐渐提上研究日程.

化学链合成氨技术作为实现 “绿色” 合成氨的可能 方案之一, 方兴未艾. 化学链合成氨技术走向应用有赖 于催化化学、材料科学、化学工程、计算化学等多学科 交叉. 随着化学链合成氨日益受到研究者的关注, 这一 技术在氨的绿色合成及可再生能源的储存与转化过程 中或将发挥重要的作用.

\section{作者简介}

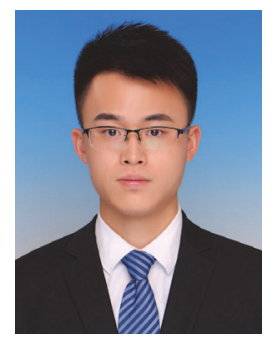

冯圣, 博士研究生. 2019 年起就读于大连理工大学张大显 化学学院攻读博士学位. 目前主要研究方向为化学链合成氨 以及电化学合成氨.

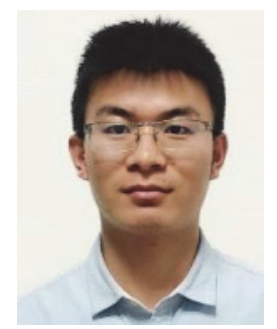

高文波, 博士. 2019 年毕业于中国科学院大连化学物理研 究所, 获理学博士学位. 主要从事催化及化学链合成氨研究.

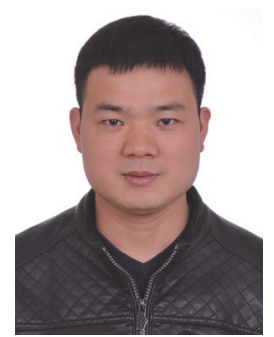

曹湖军, 副研究员, 中国科学院青年创新促进会会员. 2014 年毕业于大连理工大学, 获博士学位. 2014 年至 2018 年 
在德国亥姆霍兹材料与海洋研究所从事博士后工作. 研究方 向为新型功能化金属氢化物的合成及应用.

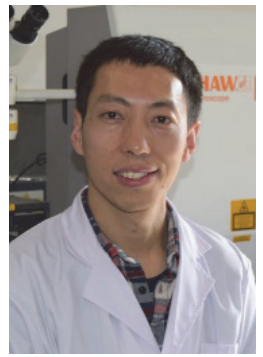

郭建平, 研究员, 博士生导师. 2015 年毕业于中国科学院 大连化学物理研究所, 获博士学位. 主要从事 $\mathrm{N}_{2} 、 \mathrm{H}_{2} 、 \mathrm{NH}_{3}$ 等小分子的活化与转化方面的研究.

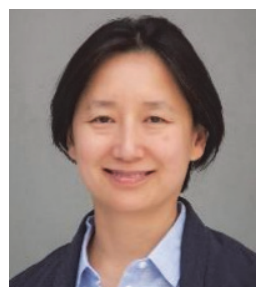

陈萍, 研究员, 博士生导师, 中国科学院大连化学物理研 究所氢能与先进材料研究部部长. 1997 年毕业于厦门大学, 获 博士学位. 研究领域主要包括储氢与多相催化方面的材料设 计与开发.

\section{References}

[1] Liu, H. Z. Chem. Ind. Eng. Prog. 2013, 32, 1995 (in Chinese). (刘化 章, 化工进展, 2013, 32, 1995.)

[2] Klerke, A.; Christensen, C. H.; Norskov, J. K.; Vegge, T. J. Mater. Chem. 2008, 18, 2304.

[3] Guo, J. P.; Chen, P. Chem 2017, 3, 709.

[4] Valera-Medina, A.; Xiao, H.; Owen-Jones, M.; David, W. I. F.; Bowen, P. J. Prog. Energy Combust. Sci. 2018, 69, 63.

[5] Smith, C.; Hill, A. K.; Torrente-Murciano, L. Energy Environ. Sci. 2020, 13,331

[6] Erisman, J. W.; Sutton, M. A.; Galloway, J.; Klimont, Z.; Winiwarter, W. Nat. Geosci. 2008, 1, 636.

[7] Wang, Q. R.; Guo, J. P.; Chen, P. J. Energy Chem. 2019, 36, 25 (in Chinese). (王倩茹, 郭建平, 陈萍, 能源化学, 2019, 36, 25.)

[8] Norskov, J. K.; Chen, J. G. Sustainable Ammonia Synthesis, US Department of Energy, 2016.

[9] Zeng, L.; Cheng, Z.; Fan, J. A.; Fan, L. S.; Gong, J. L. Nat. Rev. Chem. 2018, 2, 349.

[10] Gao, W. B.; Guo, J. P.; Wang, P. K.; Wang, Q. R.; Chang, F.; Pei, Q. J.; Zhang, W. J.; Liu, L.; Chen, P. Nat. Energy 2018, 3, 1067.

[11] Koerts, T.; Vansanten, R. A. J. C. S. Chem. Commun. 1991, 1281.

[12] Wang, Q. R.; Guo, J. P.; Chen, P. Joule 2020, 4, 705.

[13] Zeng, L.; Luo, S. W.; Li, F. X.; Fan, L. S. Sci. China Chem. 2012, 42, 260 (in Chinese). (曾亮, 罗四维, 李繁星, 范良士, 中国科学: 化学, 2012, 42, 260.)

[14] Chen, S.; Zeng, L.; Mu, R. T.; Xiong, C. Y.; Zhao, Z. J.; Zhao, C. J.; Pei, C. L.; Peng, L. M.; Luo, J.; Fan, L. S.; Gong, J. L. J. Am. Chem. Soc. 2019, 141,18653 .

[15] Gao, Y. F.; Wang, X. J.; Liu, J. C.; Huang, C. D.; Zhao, K.; Zhao, Z. L.; Wang, X. D.; Li, F. X. Sci. Adv. 2020, 6, eaaz9339.

[16] Tomkins, P.; Ranocchiari, M.; van Bokhoven, J. A. Acc. Chem. Res. 2017, 50,418

[17] Groothaert, M. H.; Smeets, P. J.; Sels, B. F.; Jacobs, P. A.; Schoonheydt, R. A. J. Am. Chem. Soc. 2005, 127, 1394.

[18] Cheng, Z.; Baser, D. S.; Nadgouda, S. G.; Qin, L.; Fan, J. A.; Fan, L. S. ACS Energy Lett. 2018, 3, 1730.

[19] Huang, C. D.; Wu, J.; Chen, Y. T.; Tian, M.; Rykov, A. I.; Hou, B.
L.; Lin, J.; Chang, C. R.; Pan, X. L.; Wang, J. H.; Wang, A. Q.; Wang, X. D. Commun. Chem. 2018, 1, 55.

[20] Liu, Y.; Qin, L.; Cheng, Z.; Goetze, J. W.; Kong, F. H.; Fan, J. A.; Fan, L. S. Nat. Commun. 2019, 10, 6.

[21] Xu, B. J.; Bhawe, Y.; Davis, M. E. Proc. Natl. Acad. Sci. U. S. A. 2012, 109, 9260.

[22] Abanades, S.; Flamant, G. Solar Energy 2006, 80, 1611.

[23] Zhu, X.; Imtiaz, Q.; Donat, F.; Muller, C. R.; Li, F. X. Energy Environ. Sci. 2020, 13, 772.

[24] Duan, Y. F.; Chen, C. Z.; Zhang, J. S.; Wang, X. H.; Wei, J. J. Sci. China Chem. 2020, 50, 337 (in Chinese). (段一菲, 陈存壮, 张军 社, 王新赫, 魏进家, 中国科学: 化学, 2020, 50, 337.)

[25] Jennings, J. R. Catalytic ammonia synthesis: Fundamentals and practice, Plenum Press, New York, 1991.

[26] Frank, A. R. Trans. Faraday Soc. 1908, 4, 099

[27] Michalsky, R.; Pfromm, P. H. AlChE J. 2012, 58, 3203.

[28] Haber, F.; van Oordt, G. Z. Anorg. Chem. 1905, 44, 341.

[29] Chen, J. G.; Crooks, R. M.; Seefeldt, L. C.; Bren, K. L.; Bullock, R. M.; Darensbourg, M. Y.; Holland, P. L.; Hoffman, B.; Janik, M. J.; Jones, A. K.; Kanatzidis, M. G.; King, P.; Lancaster, K. M.; Lymar, S. V.; Pfromm, P.; Schneider, W. F.; Schrock, R. R. Science 2018 360 , eaar6611.

[30] Galvez, M. E.; Halmann, M.; Steinfeld, A. Ind. Eng. Chem. Res. 2007, 46, 2042.

[31] Galvez, M. E.; Frei, A.; Halmann, M.; Steinfeld, A. Ind. Eng. Chem. Res. 2007, 46, 2047.

[32] Molisani, A. L.; Yoshimura, H. N. Mater. Res. Bull. 2010, 45, 733.

[33] Wu, Y.; Jiang, G. D.; Zhang, H. B.; Sun, Z.; Gao, Y.; Chen, X. P.; Liu, H. Z.; Tian, H. J.; Lai, Q. H.; Fan, M. H.; Liu, D. Chem. Commun. 2017, 53, 10664.

[34] Gao, Y.; Wu, Y.; Zhang, Q.; Chen, X. P.; Jiang, G. D.; Liu, D. Int. J. Hydrogen Energy 2018, 43, 16589.

[35] Wu, Y.; Gao, Y.; Zhang, Q.; Cai, T.; Chen, X.; Liu, D.; Fan, M. Fuel 2020, 264, 116821.

[36] Zhang, Q.; Wu, Y.; Gao, Y.; Chen, X.; Liu, D.; Fan, M. Int. J. Hydrogen Energy 2020, 45, 9903.

[37] Michalsky, R.; Pfromm, P. H. Solar Energy 2011, 85, 2642.

[38] Michalsky, R.; Pfromm, P. H. J. Phys. Chem. C 2012, 116, 23243.

[39] Michalsky, R.; Parman, B. J.; Amanor-Boadu, V.; Pfromm, P. H. Energy 2012, 42, 251.

[40] Heidlage, M. G.; Kezar, E. A.; Snow, K. C.; Pfromm, P. H. Ind. Eng. Chem. Res. 2017, 56, 14014.

[41] Medford, A. J.; Vojvodic, A.; Hummelshoj, J. S.; Voss, J.; Abild-Pedersen, F.; Studt, F.; Bligaard, T.; Nilsson, A.; Norskov, J. K. J. Catal. 2015, 328, 36.

[42] Appl, M. Ammonia: Principles and industrial practice, Wiley-VCH, Weinheim, 1999.

[43] Michalsky, R.; Avram, A. M.; Peterson, B. A.; Pfromm, P. H.; Peterson, A. A. Chem. Sci. 2015, 6, 3965.

[44] Laassiri, S.; Zeinalipour-Yazdi, C. D.; Catlow, C. R. A.; Hargreaves, J. S. J. Appl. Catal. B 2018, 223, 60.

[45] Michalsky, R.; Pfromm, P. H.; Steinfeld, A. Interface Focus 2015, 5, 20140084.

[46] Shan, N.; Chikan, V.; Pfromm, P.; Liu, B. J. Phys. Chem. C 2018, 122,6109 .

[47] Shan, N. N.; Huang, C. R.; Lee, R. T.; Manavi, N.; Xu, L. B.; Chikan, V.; Pfromm, P. H.; Liu, B. Chem CatChem 2020, 12, 2233.

[48] Jacobsen, C. J. H. Chem. Commun. 2000, 1057.

[49] Jacobsen, C. J. H.; Dahl, S.; Clausen, B. S.; Bahn, S.; Logadottir, A.; Norskov, J. K. J. Am. Chem. Soc. 2001, 123, 8404.

[50] Kojima, R.; Aika, K. Chem. Lett. 2000, 514.

[51] McKay, D.; Gregory, D. H.; Hargreaves, J. S. J.; Hunter, S. M.; Sun, X. Chem. Commun. 2007, 3051

[52] Hunter, S. M.; McKay, D.; Smith, R. J.; Hargreaves, J. S. J.; Gregory, D. H. Chem. Mater. 2010, 22, 2898.

[53] Hunter, S. M.; Gregory, D. H.; Hargreaves, J. S. J.; Richard, M.; Duprez, D.; Bion, N. ACS Catal. 2013, 3, 1719.

[54] Zeinalipour-Yazdi, C. D.; Hargreaves, J. S. J.; Catlow, C. R. A. J. Phys. Chem. C 2015, 119, 28368.

[55] Alexander, A. M.; Hargreaves, J. S. J.; Mitchell, C. Top. Catal. 2012, $55,1046$.

[56] Alexander, A. M.; Hargreaves, J. S. J.; Mitchell, C. Top. Catal. 2013 $56,1963$.

[57] Roy, D.; Navarro-Vazquez, A.; Schleyer, P. V. R. J. Am. Chem. Soc. 2009, 131, 13045. 
[58] Swearer, D. F.; Knowles, N. R.; Everitt, H. O.; Halas, N. J. ACS Energy Lett. 2019, 4, 1505.

[59] McEnaney, J. M.; Singh, A. R.; Schwalbe, J. A.; Kibsgaard, J.; Lin, J. C.; Cargnello, M.; Jaramillo, T. F.; Nørskov, J. K. Energy Environ. Sci. 2017, 10, 1621.

[60] Goshome, K.; Miyaoka, H.; Yamamoto, H.; Ichikawa, T.; Ichikawa, T.; Kojima, Y. Mater. Trans. 2015, 56, 410.

[61] Yamaguchi, S.; Ichikawa, T.; Wang, Y. M.; Nakagawa, Y.; Isobe, S.; Kojima, Y.; Miyaoka, H. ACS Omega 2017, 2, 1081.

[62] Yamaguchi, T.; Shinzato, K.; Yamamoto, K.; Wang, Y.; Nakagawa, Y.; Isobe, S.; Ichikawa, T.; Miyaoka, H.; Ichikawa, T. Int. J. Hydrogen Energy 2020, 45, 6806.
[63] Gao, W. B.; Guo, J. P.; Chen, P. Chin. J. Chem. 2019, 37, 442.

[64] Veser, G. Nat. Energy 2018, 3, 1025.

[65] Hagen, S.; Barfod, R.; Fehrmann, R.; Jacobsen, C. J. H.; Teunissen, H. T.; Chorkendorff, I. J. Catal. 2003, 214, 327.

[66] Liu, T.; Temprano, I.; Jenkins, S. J.; King, D. A. J. Chem. Phys. 2013, 139, 184708

[67] Vojvodic, A.; Medford, A. J.; Studt, F.; Abild-Pedersen, F.; Khan, T. S.; Bligaard, T.; Norskov, J. K. Chem. Phys. Lett. 2014, 598, 108.

[68] Michalsky, R.; Steinfeld, A. Catal. Today 2017, 286, 124.

[69] Bartel, C. J.; Rumptz, J. R.; Weimer, A. W.; Holder, A. M.; Musgrave, C. B. ACS Appl. Mater. Interfaces 2019, 11, 24850.

(Cheng, B.) 